\title{
Analysis of warping and distortion transmission in mixed shell-GBT (generalized beam theory) models
}

\author{
Marcelo J. Bianco ${ }^{1}$ - Abinet K. Habtemariam ${ }^{1}$ - Carsten Könke ${ }^{1}$. Volkmar Zabel ${ }^{1}$
}

Received: 1 March 2018 / Accepted: 8 February 2019 / Published online: 22 February 2019

(c) The Author(s) 2019

\begin{abstract}
Warping and distortion are relevant kinematic features of thin-walled beam structures, which have a non-trivial analysis. On this basis, this paper not only evaluates the possible kinematic transmissions involving high-order warping and distortion, but also presents a procedure to analyze structures using mixed models based on shell and Generalized Beam Theory (GBT) elements. In this mixed beam-shell structure, the traditional shell elements are applied at structural detailing points, such as joints, and GBT elements are used to model the beams/columns. Such a modeling technique uses the benefits of both elements. Shell elements can easily simulate different types of geometry conditions and details, such as stiffeners and holes; meanwhile, for the beams and columns, GBT can provide high performance, accuracy, and an easy modeling approach with clear results. The numerical formulation is based on multi-freedom constraint techniques. Special attention is given to the Master-Slave method, which is developed based on GBT kinematic assumptions. Furthermore, there is a discussion concerning the choice of the master degrees of freedom and its implications in numerical performance. An example of a thin-walled hollow circular cross section illustrates the proposed approach and is compared with fully shell element models.
\end{abstract}

Keywords Generalized beam theory · Coupling equations · Structural joints · Mixed GBT-shell model · Multi-freedom constraints

\section{Introduction}

Generalized beam theory (GBT) is a numerical approach that was initially developed to describe open thin-walled beams by Richard Schardt in Darmstadt, Germany. This approach is applicable in linear analysis (Schardt 1989), but it has been further extended to geometric non-linear analysis (Schardt 1994a, b; Davies et al. 1994). As an introductory point, this theory can be understood as a generalization of Vlasov beam theory (Vlasov 1961). Beyond that, GBT has a wider range of analysis, which involves distortion and

Marcelo J. Bianco

marcelo.jose.bianco@uni-weimar.de

Abinet K. Habtemariam

abinet.habtemariam@uni-weimar.de

Carsten Könke

carsten.koenke@uni-weimar.de

Volkmar Zabel

volkmar.zabel@uni-weimar.de

1 Institute of Structural Mechanics, Bauhaus-Universitat Weimar, Weimar, Germany shear deformation (Silvestre 2003; Silvestre and Camotim 2012; de Miranda et al. 2013). In fact, GBT uses concepts of separation of variables to create (1) generalized crosssection properties, which are based on shell theory, and (2) a spatially dependent longitudinal beam function. As a result, this approach presents an astonishing numerical performance and a clear representation of the displacement and stress fields as a linear combination of the generalized cross-section properties and internal forces.

However, the majority of studies and applications of GBT are limited to a single beam model. Since many structures are composed of beam frames, it is necessary to handle the interaction among beams that share a common joint. Here, there are two obstacles that stand out. The first one is due to the complexity in the kinematic coupling among the GBT's deformation shapes at the joint, which involves several modes simultaneously. The next section presents some examples that illustrate how complex this issue is. The second obstacle concerns the physical connection itself. In practical structural design, a substantial amount of details arises at the connection, such as stiffeners, bolts, and weldings for steel structures. These details not only change the 


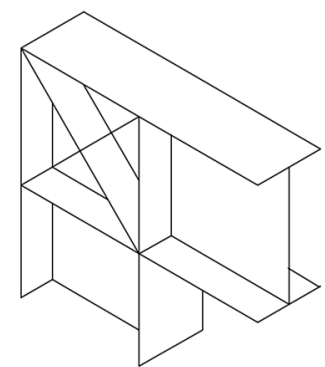

Box-Diagonal

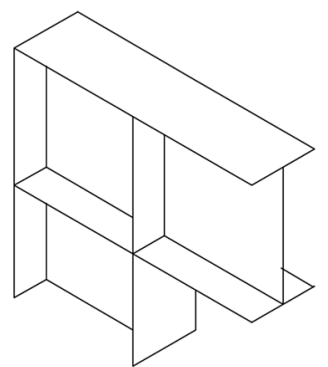

Box

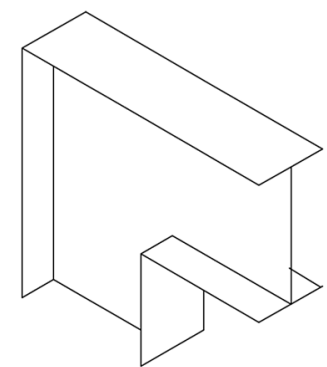

Non-Stiffener

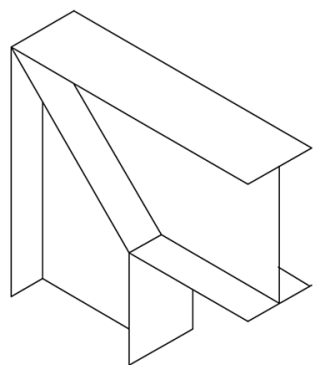

Diagonal

Fig. 1 Type of connections to clarify the kinematic behavior in high modes

kinematic behavior of the connection, but also cannot be modeled as beam elements.

Thus, due to the high level of details in structural connections, shell and solid elements are widely applied in their structural analysis. Therefore, it is convenient to split the structural analysis into two domains: the first is the beam domain, in which GBT elements are applied; the second one is the connection domain, in which shell or solid elements are used.

This domain separation leads directly to the question of how to couple the degrees of freedom of shell and solid elements with the high order of GBT's degrees of freedom, which represent the modal shape deformations.

The current study develops a novel application of multifreedom constraints in a coupling method between shell and GBT elements to solve the issue of the previous paragraph. In addition, it provides a detailed numerical example to illustrate the proposed method, as well as compare the result of the mixed GBT-shell models with those of pure shell models.

\section{Kinematics in warping and distortion transmission}

Structural joints are good examples of how complex the kinematics of warping and distortion transmission are, especially if a structural frame is modeled only by beam elements.

The first studies in this problem were not directly related to GBT, but they were concerned with the warping transmission of a Vlasov beam element (Fujitani 1998; Tong et al. 2005; Kindmann and Kraus 2011), which can be understood as the first high mode of warping in GBT.

Basaglia et al. $(2008,2012)$ were the first ones to study the warping as a high mode of GBT, which highlighted not only the main role of the layout of connection in warping transmission, but also identified and classified the extrakinematic behavior of connection in high modes: internal support (no internal force and deformation transmission) and inverse-clamp (totally inverse transmission of internal forces and deformation).

To clarify how the connection layout affects the warping and distortion transmission, one can consider four particular types of connections between beams with an "I" profile: box-diagonal, box, non-stiffener and diagonal connections, as shown in Fig. 1. In fact, these types of welded steel connections are widely applied in structural design. Each one of these connections is detailed in sequence.

\section{Internal support mechanism}

The connection that leads to an internal support behavior is the box-diagonal one. Here, the internal support mechanism is nothing more than the restraint of cross-section warping and/or distortion by stiffeners. Since all internal forces, which are beyond the traditional six degrees of freedom, have two or more opposite forces and/or bending moments in the cross-section, a stiffener component can directly link these opposite forces and/or bending moments, which will nullify them.

For instance, in the particular case of non-uniform torsion of Vlasov, the seventh degree of freedom indicates warping in the node. Different from the six traditional degrees of freedom, which can be understood as a representation of rigid-body movement at one node, the seventh degree of freedom indicates deformation at the node itself.

Following this representation, a node cannot be interpreted as a point but as an elementary volume, as shown by the elementary cube in Fig. 2. ${ }^{1}$ Consequently, the warping degree of freedom, and any other GBT's high deformation

\footnotetext{
1 The figure also presents the unique behavior of high modes. In the case of torsional warping, a body with positive warping in the $\mathrm{x}$ axis also can be understood as an increase of rotation around the same $\mathrm{x}$ axis in the $\mathrm{x}$ axis direction. However, it can also be described as a combination of simultaneous negative warping in the $\mathrm{y}$ and $\mathrm{z}$ directions.
} 


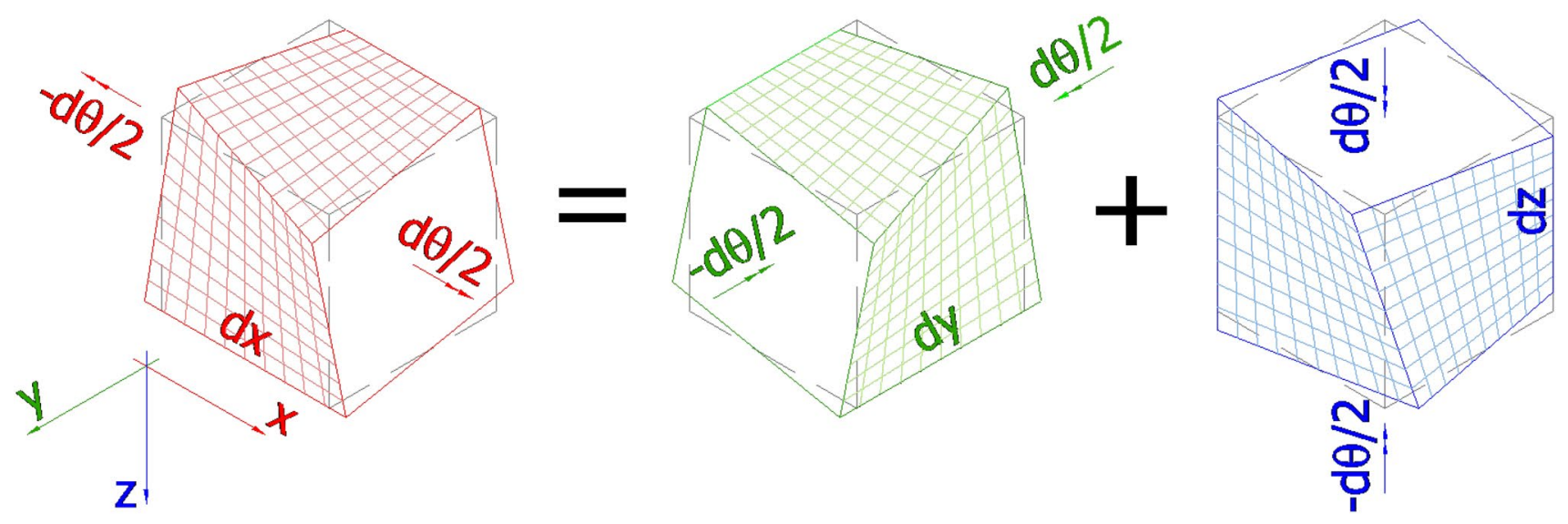

Fig. 2 Elementary node to describe the kinematic behavior of the connection, which involves warping

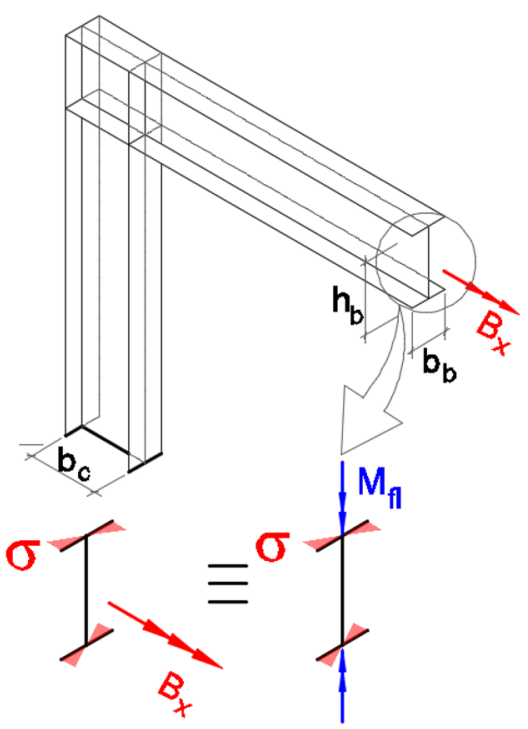

(a)

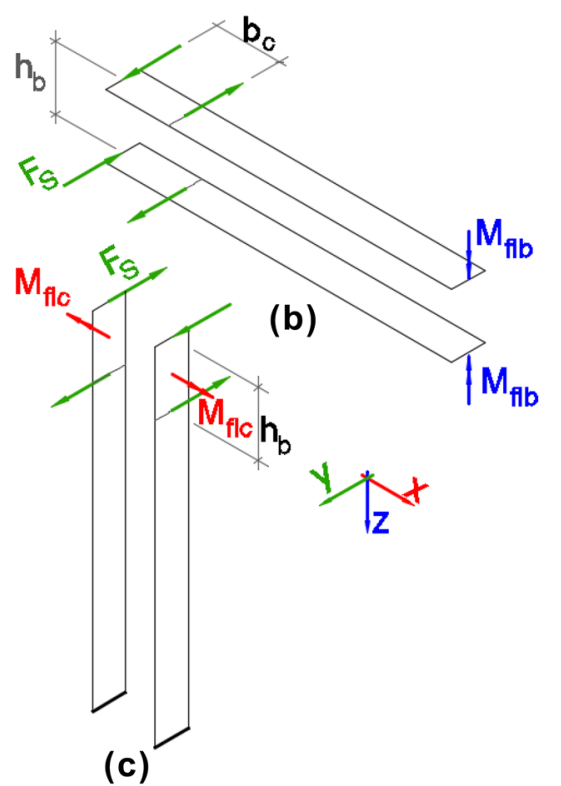

(c)

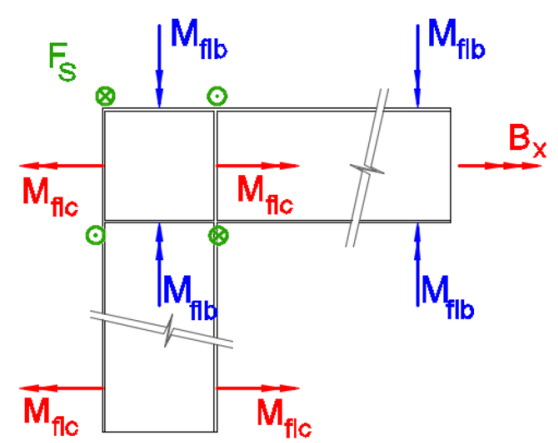

(d)

Fig. 3 Kinematic mechanism of box connection; a bi-moment decomposition; $\mathbf{b}$ and $\mathbf{c}$ local bending moments in the flanges of the beam and column; $\mathbf{d}$ resume of the kinematic interpretation

mode, can be understood as the nodal deformation mode, i.e., the warping is only one of several possible deformations of the high modes at the node.

The remarkable property of any degree of freedom higher than the six of rigid-body movement is that it does not necessarily need an external restriction (such as building foundations) to have a support conditional. In other words, it is possible to have a support for the node's deformation only by stiffening the node. Therefore, the internal support is a node that is stiff enough to avoid the warping or distortion deformation, such as the box-diagonal connection, illustrated above.

\section{Inverse-clamp mechanism}

To explain how it is possible to transmit inverse internal forces of high modes, one can consider the simple beamcolumn frame under an external bi-moment, as presented in Fig. 3.

According to Vlasov's assumption that the cross section does not remain plane in the deformation configuration, each flange can be interpreted as a single beam. Hence, the flanges can rotate independently from each other due to the low stiffness of the web. 

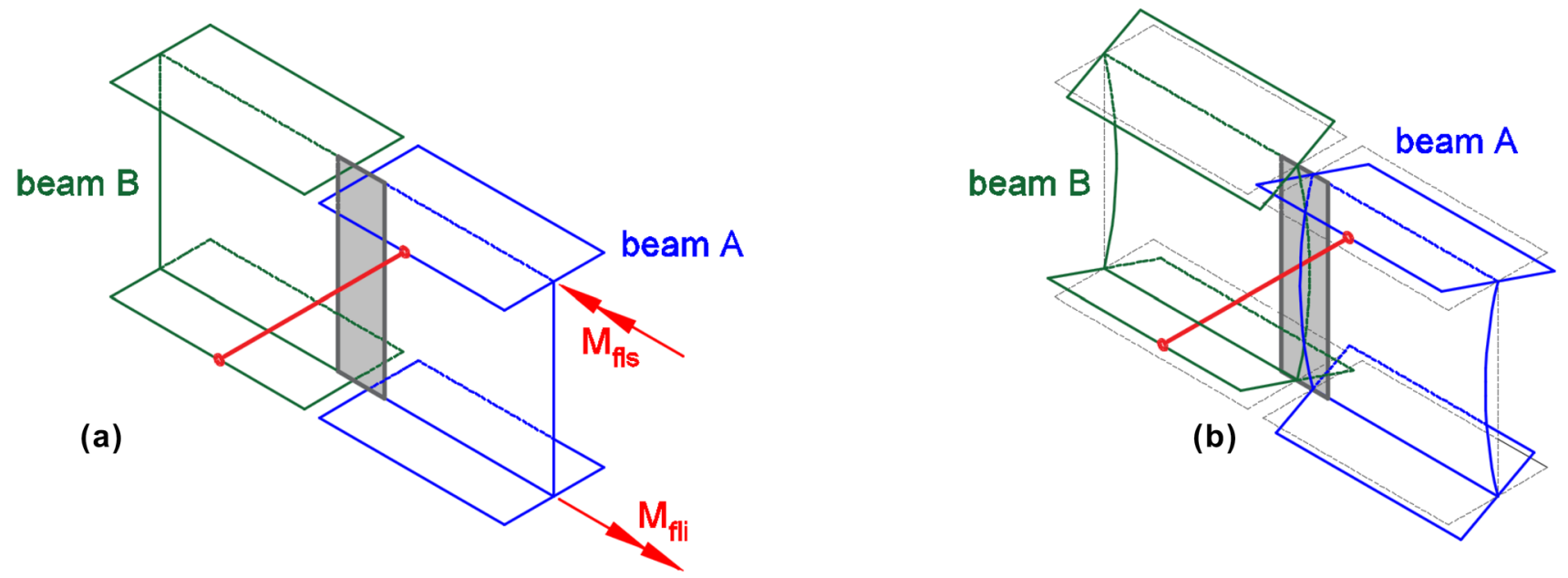

Fig. 4 Kinematic mechanism of inverse distortion transmission; a undeformed structure under a bi-torsion internal force (the connection rod is in red); $\mathbf{b}$ deformed structure with inverse transmission

Decoupling the upper and lower flanges from the structure shown in Fig. 3, one obtains two cantilever beams. Their supports are the two vertical stiffeners of the box connection, which leads to a binary reaction force. Since the vertical stiffeners have low values of torsion stiffness, each vertical stiffener acts as hinge support.

The reaction forces in the stiffeners, with the principle of action and reaction, act in the column's flanges as concentrated bending moments, similar to the case of the beam's flanges. However, these local bending moments in the column have an opposite direction compared to the local bending moments in the flange of the beam. For instance, in Fig. 3, the beam's moment vectors of the flanges are inward oriented, while, in the column, these vectors are outward oriented.

It is important to note that this unique property of warping transmission also exists in the other high modes. As a second example, one can consider the following "I" beam under an opposite dual self-balanced torsion moment, ${ }^{2}$ as illustrated in Fig. 4. In this example, the upper flange's extreme of beam A is linked by a rigid rod to the bottom flange's extreme of beam B. Since the rod creates a compatibility of displacement between these flanges, the vertical displacement of the upper flange will be the same as that of the bottom flange. However, the vertical displacement of the bottom flange's extreme in beam B is opposite that of the same point in beam A. Thus, the total distortion of beam B is opposite that of beam A. Despite the fact that this connection is not usual in current structures, it is an example of how the inverse transmission of distortion can be produced.

\footnotetext{
2 The dual self-balanced torsion moment is named here as bi-torsion in analogy to the Vlasov bi-moment.
}

\section{Coupling of warping and distortion due to connections}

The non-stiffener connection not only explains the hinge behavior of GBT's high modes, but also is a good example of how warping and distortion can be coupled due to the connection.

Similar to the explanation of box connection, Fig. 5 shows how the flanges of the beam can be treated as cantilever beams. Furthermore, the cross-section bi-moment can be expressed as a local bending moment of these cantilever beams.

However, in the case of non-stiffener connection, there is no reaction binary force composed by the vertical stiffener. The only restraints provided are the torsional inertia of each individual column's flange and the flexural bending stiffness of the web. Both effects in general cases have low values compared to the moment of inertia of the beam's flange.

Consequently, only residual warping is transmitted among the elements. If the thicknesses of the web and the column's flange tend to zero, then the non-stiffener connection can be considered as an ideal warping hinge connection.

It is important to note two effects in this connection type. The first one is the inversion of the residual bi-moment from the beam into the column. This inversion is due to the web's warping transmission, which can be understood as an elementary slice of the cube presented in Fig. 2. Naturally, the web capacity for warping transmission is quite limited.

The second effect is the coupling of the residual transmitted warping with the distortion of the column's cross section. The non-stiffener connection is an emblematic example of how the warping is coupled with distortion. In Fig. 5b, c, a kinematic interpretation of how the local bending moments in the flange become local torsion moments in the column's 


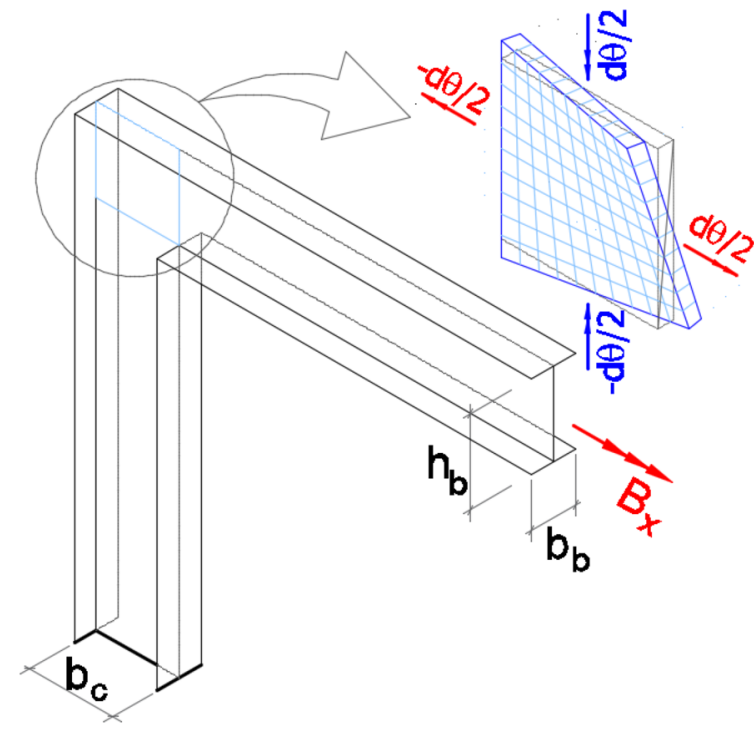

(a)

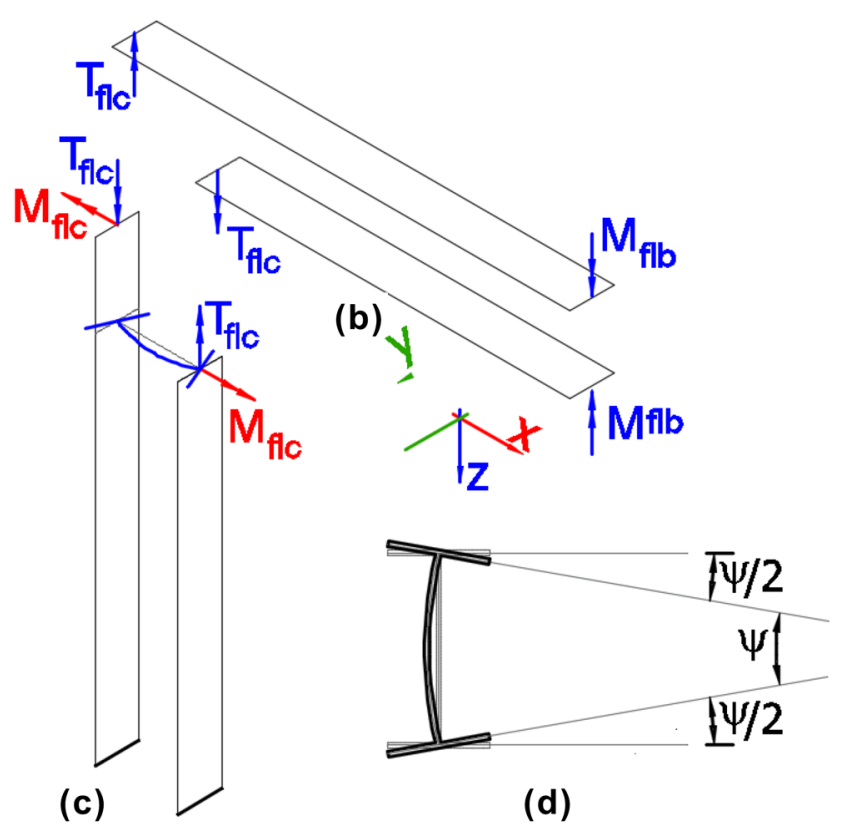

(d)

Fig. 5 Kinematic mechanism of non-stiffener connection; $\mathbf{a}$ warping deformation of the web; $\mathbf{b}$ and $\mathbf{c}$ local torsional and bending moments in the flanges of the beam and column; d distortion shape in the column's cross section

flange is shown. These torsion moments are self-balanced in the cross section, which can be described as bi-torsion. This distortion-torsional mode has no warping stiffness. Therefore, this mode only involves the transversal bending and shear stiffness matrices of the cross section.

\section{Clamp mechanism}

The clamp mechanism behavior of high modes can be explained by the diagonal connection. The kinematic behavior of this connection, which does not invert the high modes' internal forces and displacements, can be understood based on the equilibrium of the flanges and the stiffener.

Different from the box connections, the local bending moments of the beam's flanges are not balanced by binary forces due to the reactions of the stiffeners. Instead, they are balanced by the moments' equilibrium at intersection points among (1) the flanges of the column; (2) the flange of the beam; and (3) the diagonal stiffener, as the point " $\mathrm{A}$ " presented in Fig. 6.

Analogous to the case of trusses, point A in Fig. $6 \mathrm{~b}, \mathrm{c}$ has its equilibrium when the sum of the moment's vector is null. Naturally, the only difference with respect to the vectorial equilibrium approach in trusses is that the bending vectors are always perpendicular to the flanges and the stiffeners and not aligned with them.

Following this idea and by the third Newton's law, the local bending moments in the column's flanges have an "incoming" direction, such as the local bending moments in the beam's flanges. Therefore, there is no inversion of the warping and bi-moment direction.

However, this simple explanation has the assumption of null torsional inertia in the stiffener and in the flanges. If the connection has a thick stiffener and flanges, then the torsion moment vector, which is aligned with the stiffeners and flanges, will restrain the connection to warping. In other words, as perpendicular vectors with respect to the bending moment vectors, the torsional vectors will also restrain the connection in the stiffener's direction and not only in the perpendicular direction with respect to it as a "membrane" diagonal.

To clarify this, as shown in Fig. 7, the torsion moments can be understood as bending moments in "virtual stiffener and flanges," which are fully perpendicular to the real one. Then, a simple diagonal connection becomes a box-diagonal, which is an internal support condition, as explained before.

The final remark about this connection is the effect of the residual web's warping transmission. As explained in the previous subsection, the web has an inverse warping transmission, as well as a box connection.

In fact, the diagonal connection is a clear example of how complex a connection's kinematic model can reach. Even more complex issues arise in the generic cross section, in which the centers of gravity and shear are not coincident at the same point. 


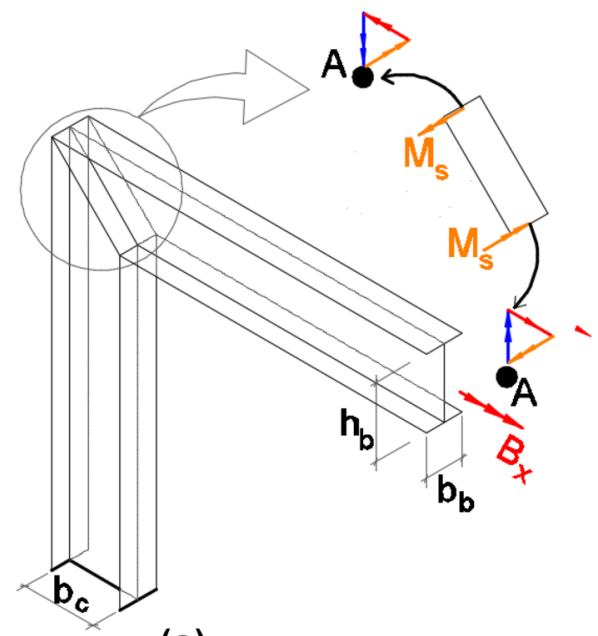

(a)

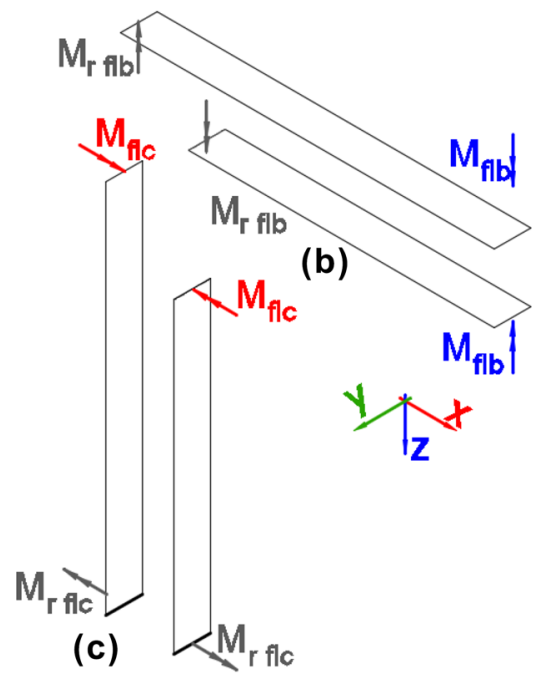

(c)

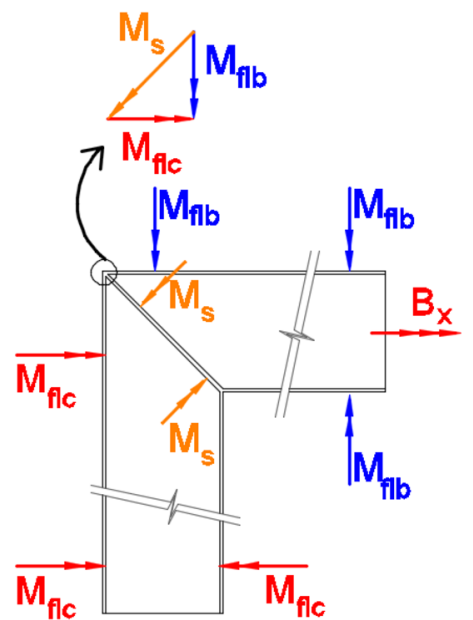

(d)

Fig. 6 Kinematic mechanism of diagonal connection; a bi-moment equilibrium as nodal equilibrium; $\mathbf{b}$ and $\mathbf{c}$ bending moments in the stiffener and in the flanges of the beam and column; $\mathbf{d}$ left view of the nodal moment equilibrium

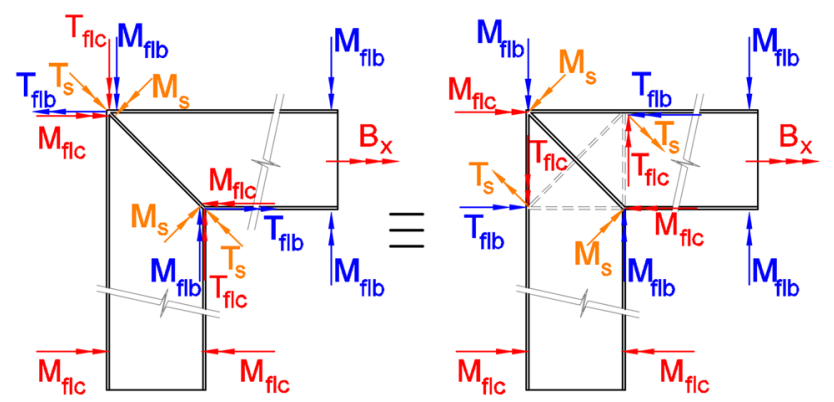

Fig. 7 Diagonal connection with the effects of torsion inertia of flanges and stiffener resulting in a box-diagonal connection

\section{Coupling between GBT and shell elements}

From the examples of warping transmission in the previous section, it becomes clear that the connections involving GBT's high modes are not only complex in the kinematic mechanism but also in the unique properties of these modes. In addition, a usual structure connection involves many details, such as welds, bolts, holes, and stiffeners, which require minute modeling and analysis, usually carried out by shell or solid finite elements.

Since the main field of application of GBT is thinwalled structures and the connections of these structures are often modeled by shell elements, the focus of this section is on the coupling between these elements and GBT elements.

The coupling approach proposed here is a novel application of Master-Slave method for beam frames and connections (Jelenić and Crisfield 1996; Munoz et al. 2003).
It uses the superposition property of GBT's deformation modes to set up the relationship between the master and slave degrees of freedom. Furthermore, these degrees of freedom are divided in two groups: shell and GBT. Each group is related to a master degree or slave degree of freedom. Different from the traditional applications, the presented approach predefines which group is the master and which is the slave. These details are discussed in sequence.

\section{Definition of master and slave degrees of freedom}

Usually, in the Master-Slave multi-freedom constraint method, there is no rule regarding the definition of which degree of freedom is the master and which one is the slave. However, in the present implementation, this definition is predefined. Since the main focus of the proposed procedure is to build up a degree of freedom mapping of type shell-GBT or GBT-shell and avoid any type of restraint of type GBT-GBT or shell-shell, one can classify the degree of freedom as shell or GBT group. Once this limitation and classification are defined, the next issue is which group is the master and which is the slave.

The direct answer to this question is that the degrees of freedom of GBT are the master ones.

This choice is not arbitrary. It is based on the fact that one GBT node has a lower amount of degrees of freedom than the summation of all degrees of freedom of shell elements used to described a cross section. For instance, one can consider the coupling of a hollow circular cross section, as plotted in Fig. 8. This coupled cross section has 28 shell nodes; each node has 6 degrees of freedom, giving a total of 168 degrees of freedom. This same cross-section can be described in GBT by 6 deformation modes, each one having 


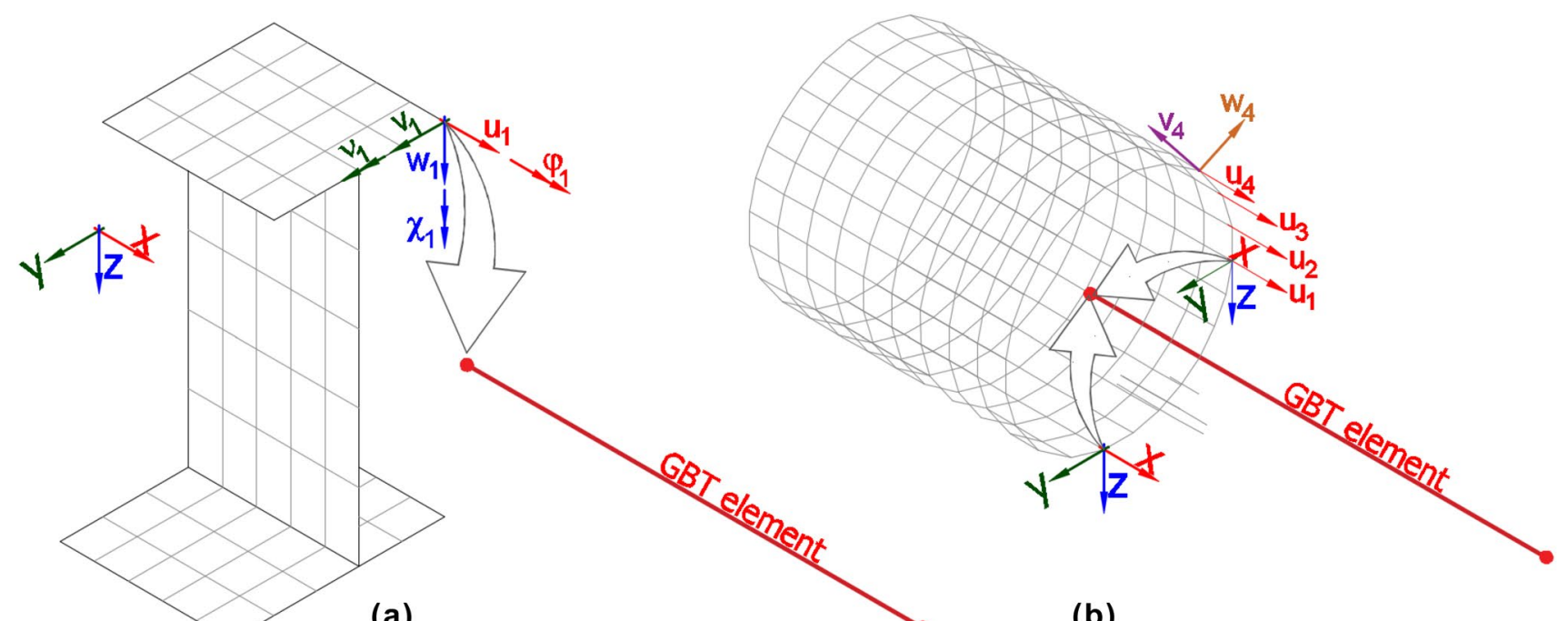

(a)

(b)

Fig. 8 Orientation of the degrees of freedom in the coupling between shell and GBT elements: a generic-segmented cross section; b a hollow circular cross section

two degrees of freedom per node (there are a few exceptions, such as the uniform torsion or the uniform longitudinal elongation deformation modes, which require just one degree of freedom per node). Consequently, GBT requires a total of 12 degrees of freedom.

Since the finite-element model's response is always limited by the coarse description of the cross section, the opposite choice, i.e., the shell's degrees of freedom as master ones, is numerically inefficient. This alternative definition requires more computational effort to reach the results of the choice mentioned in the previous paragraph.

\section{Master-Slave relationships based on GBT's deformation modes}

The coupling between shell and GBT degrees of freedom can be expressed in matrix form as follows:

$[\vartheta]_{\text {shell }}=\left[T_{c}\right][\vartheta]_{\mathrm{GBT}}$,

where $[\vartheta]_{\text {shell }}$ and $[\vartheta]_{\mathrm{GBT}}$ are the displacement vectors of shell and GBT degrees of freedom, respectively, and $\left[T_{c}\right]$ is the multi-freedom constraint transformation matrix, which is based on GBT's description of the displacement field as a summation of the modal displacements:

$u(x, s)=\sum_{i=1}^{n}{ }^{i} u(s){ }^{i} V_{, x}(x)$
$v(x, s)=\sum_{i=1}^{n}{ }^{i} v(s)^{i} V(x)$ $w(x, s)=\sum_{i=1}^{n}{ }^{i} w(s)^{i} V(x)$.

Here, $u, v$, and $w$ are the displacements in the longitudinal, transversal tangential, and transversal perpendicular directions, respectively, as shown in Fig. 8; $V$ is the amplification function of these displacements along the beam length; and the upper left index $i$ indicates GBT's deformation mode. Hence, these equations are used to express the shell's nodal displacement. For instance, the longitudinal displacement of node 1, in the shell element discretization shown in Fig. 8, can be expressed as follows:

$u_{1, \text { shell }}=\sum_{i=1}^{m}{ }^{i} u(s){ }^{i} V_{, x}(x=-L / 2)$,

where the longitudinal position in the beam element, $x$, is set at one of the extreme nodes: $x=-L / 2$ or $x=L / 2$ (in a centered-origin element). This summation can be represented in a matrix form, which is already the main component of the multi-freedom constraint transformation matrix:

$$
[u]_{\mathrm{shell}}=\left[T_{c}\right]_{u}\left[V_{, x}\right]_{\mathrm{GBT}}
$$

$\left[\begin{array}{c}u_{1} \\ u_{2} \\ u_{3} \\ u_{4} \\ \vdots \\ u_{n}\end{array}\right]_{\text {shell }}=\left[\begin{array}{ccccc}{ }^{1} u_{1} & { }^{2} u_{1} & { }^{3} u_{1} & \ldots & { }^{m} u_{1} \\ { }^{1} u_{2} & { }^{2} u_{2} & { }^{3} u_{2} & \ldots & { }^{m} u_{2} \\ { }^{1} u_{3} & { }^{2} u_{3} & { }^{3} u_{3} & \ldots & { }^{m} u_{3} \\ { }^{1} u_{4} & 2 u_{4} & { }^{3} u_{4} & \ldots & { }^{m} u_{4} \\ \vdots & \vdots & \vdots & \ddots & \vdots \\ { }^{1} u_{n} & 2 u_{n} & { }^{3} u_{n} & \ldots & { }^{m} u_{n}\end{array}\right]\left[\begin{array}{c}{ }^{1} V \\ { }^{2} V_{, x} \\ { }^{3} V_{, x} \\ \vdots \\ { }^{m} V_{, x}\end{array}\right]_{\mathrm{GBT}}$ 
Here, the indexes $n$ and $m$ indicate the number of shell nodes and GBT modes, respectively.

It is not necessary that the shell nodes and the GBT crosssection discretization nodes are in the same geometric position. The only restriction is the imposition that shell nodes have to be in the middle line of the cross-section walls. Since this condition is maintained, an arbitrary shell node position can be coupled with GBT modes by the interpolation functions of the cross-section segment. For instance, a linear interpolation function of $u$, in a centered-origin segment of a cross-section, can be expressed by the following:

$$
\begin{aligned}
{ }^{i} u_{h}(s) & =\left[\begin{array}{ll}
s & 1
\end{array}\right] \frac{1}{2 l_{s}}\left[\begin{array}{cc}
-2 & 2 \\
l_{s} & l_{s}
\end{array}\right]^{i}\left[\begin{array}{l}
u_{1} \\
u_{2}
\end{array}\right] \\
& =\left[T s_{H e 1}\right]\left[S h_{H e 1 c c}\right]^{i}\left[u_{h}\right],
\end{aligned}
$$

where $s$ is the position of the shell node in the cross-section segment; $l_{s}$ is the total segment length; $\left[T_{H e 1}\right]$ is the vector with polynomial terms; $\left[S h_{H e 1 c c}\right]$ is the matrix with the coefficients of completeness requirements for Hermitian shape functions. The introduction of this expression into Eq. 6 leads to the following equation:

$$
\left[\begin{array}{c}
u_{1} \\
u_{2} \\
u_{3} \\
u_{4} \\
\vdots \\
u_{n}
\end{array}\right]_{\text {shell }}=[T S 1]_{\text {seg }}\left[u_{h}\right]_{\text {seg }}\left[\begin{array}{c}
{ }^{1} V \\
{ }^{2} V_{, x} \\
{ }^{3} V_{, x} \\
\vdots \\
{ }^{n} V_{, x}
\end{array}\right]_{\mathrm{GBT}} .
$$

Here, $[T S 1]_{\text {seg }}$ is a matrix, in which the number of rows is the number of shell nodes and the number of columns is the number of segments of the cross-section that contains the shell nodes. This matrix is populated in each row by the linear Hermitian interpolation function of $\left[T_{H_{e l}}\right]\left[S h_{H e 1 c c}\right]$ in the respective column of the segment that has the shell node.

For example, in the coupling illustrated in Fig. 9, the shell node 1 is in cross-section segment 1 , shell nodes 2 and 3 are in segment 2 , and shell node 4 is in segment 3 . For this case, one obtains the following interpolation segment matrix:

$[T S 1]_{\text {seg }}=\left[\begin{array}{cccc}T S_{1} & 0 & 0 & \ldots \\ 0 & T S_{2} & 0 & \ldots \\ 0 & T S_{2} & 0 & \ldots \\ 0 & 0 & T S_{3} & \ldots \\ \vdots & \vdots & \vdots & \ddots\end{array}\right]$

where $T S_{i}$ is the product of $\left[T s_{H e 1}\right]_{s, i}\left[S h_{H e 1 c c}\right]_{s, i}$ for each $i$ cross-section segment and the lower right indexes $s 1, s 2$, and $s 3$ indicate the relative cross-section segment. The second

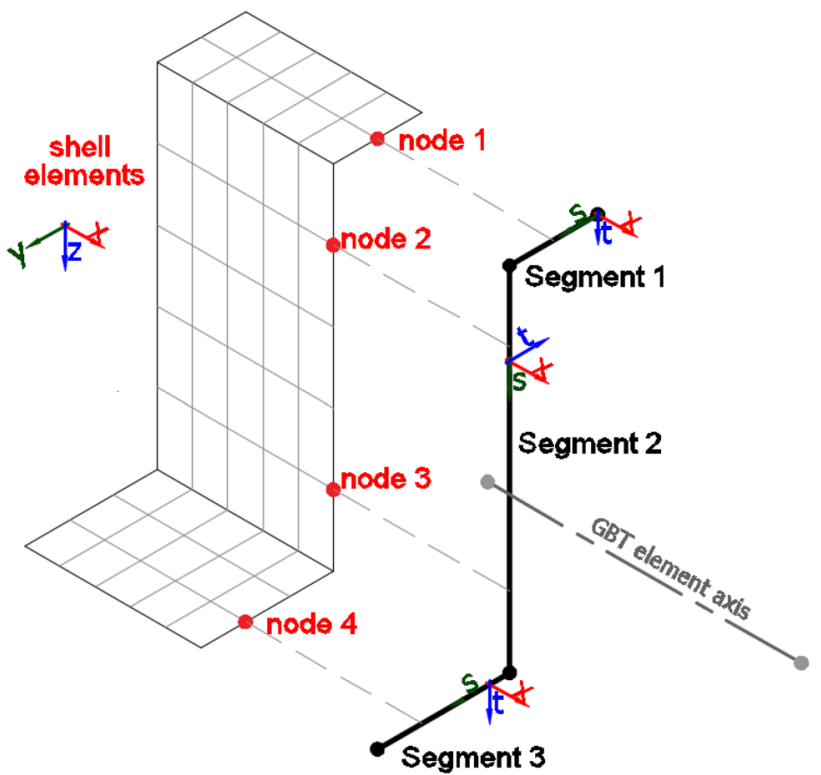

Fig. 9 Example of coupling of shell nodes in the middle of GBT's cross-section segments

matrix in the right-hand side of Eq. $8,\left[u_{h}\right]_{\text {seg }}$, is nothing more than all of the modal longitudinal displacements of each segment that has the shell nodes:

$\left[u_{h}\right]_{\text {seg }}=\left[\begin{array}{ccccc}{ }^{1}\left[u_{h}\right]_{s 1} & { }^{2}\left[u_{h}\right]_{s 1} & 3\left[u_{h}\right]_{s 1} & \ldots & m\left[u_{h}\right]_{s 1} \\ 1\left[u_{h}\right]_{s 2} & 2\left[u_{h}\right]_{s 2} & 3\left[u_{h}\right]_{s 2} & \ldots & m\left[u_{h}\right]_{s 2} \\ 1\left[u_{h}\right]_{s 3} & 2\left[u_{h}\right]_{s 3} & 3\left[u_{h}\right]_{s 3} & \ldots & m\left[u_{h}\right]_{s 3} \\ \vdots & \vdots & \vdots & \ddots & \vdots\end{array}\right]$

The transversal displacements $v$ and $w$ are obtained in a similar way. The only change is the absence of mode 1 (axial deformation). If the shell nodes share the same position as the GBT nodes of cross-section discretization, one finds the following:

$$
\begin{aligned}
& {[v]_{\text {shell }} }=\left[T_{c}\right]_{v}[V]_{\mathrm{GBT}} ; \\
& {\left[\begin{array}{c}
v_{1} \\
v_{2} \\
v_{3} \\
\vdots \\
v_{n}
\end{array}\right]_{\text {shell }}=\left[\begin{array}{ccccc}
{ }^{2} v_{1} & { }^{3} v_{1} & { }^{4} v_{1} & \ldots & { }^{n} v_{1} \\
{ }^{2} v_{2} & { }^{3} v_{2} & { }^{4} v_{2} & \ldots & { }^{n} v_{2} \\
{ }^{2} v_{3} & { }^{3} v_{3} & { }^{4} v_{3} & \ldots & { }^{n} v_{3} \\
\vdots & \vdots & \vdots & \ddots & \vdots \\
{ }^{2} v_{6} & { }^{3} v_{6} & { }^{4} v_{6} & \ldots & { }^{n} v_{6}
\end{array}\right]\left[\begin{array}{c}
{ }^{2} V \\
{ }^{3} V \\
{ }^{4} V \\
\vdots \\
{ }^{n} V_{, x}
\end{array}\right]_{\mathrm{GBT}} }
\end{aligned}
$$

$$
\begin{aligned}
{[w]_{\text {shell }} } & =\left[T_{c}\right]_{w}[V]_{\mathrm{GBT}} ; \\
{\left[\begin{array}{c}
w_{1} \\
w_{2} \\
w_{3} \\
\vdots \\
w_{n}
\end{array}\right]_{\text {shell }} } & =\left[\begin{array}{ccccc}
{ }^{2} w_{1} & { }^{3} w_{1} & { }^{4} w_{1} & \ldots & { }^{n} w_{1} \\
{ }^{2} w_{2} & { }^{3} w_{2} & { }^{4} w_{2} & \ldots & { }^{n} w_{2} \\
{ }^{2} w_{3} & { }^{3} w_{3} & { }^{4} w_{3} & \ldots & { }^{n} w_{3} \\
\vdots & \vdots & \vdots & \ddots & \vdots \\
{ }^{2} w_{6} & { }^{3} w_{6} & { }^{4} w_{6} & \ldots & { }^{n} w_{6}
\end{array}\right]\left[\begin{array}{c}
{ }^{2} V \\
{ }^{3} V \\
{ }^{4} V \\
\vdots \\
{ }^{n} V
\end{array}\right]_{\mathrm{GBT}}
\end{aligned}
$$


Otherwise, the shell nodes are at another point of the crosssection segments, it is necessary to apply the interpolation functions of respective displacement, which leads to the following:

$\left[\begin{array}{c}v_{1} \\ v_{2} \\ v_{3} \\ v_{4} \\ \vdots \\ v_{n}\end{array}\right]_{\text {shell }}=[T S 1]_{\text {seg }}\left[v_{h}\right]_{\text {seg }}\left[\begin{array}{c}{ }^{2} V \\ { }^{3} V \\ { }^{4} V \\ \vdots \\ { }^{n} V\end{array}\right]_{\mathrm{GBT}}$

$\left[\begin{array}{c}w_{1} \\ w_{2} \\ w_{3} \\ w_{4} \\ \vdots \\ w_{n}\end{array}\right]_{\text {shell }}=[T S 3]_{\text {seg }}\left[w_{h}\right]_{\text {seg }}\left[\begin{array}{c}{ }^{2} V \\ { }^{3} V \\ { }^{4} V \\ \vdots \\ { }^{n} V\end{array}\right]_{\mathrm{GBT}}$

where $[T S 3]_{\text {seg }}$ is the cubic Hermitian shape function of transversal displacement $w$ given by the following:

$$
\begin{aligned}
{ }^{i} w_{h}(s) & \left.=\left[\begin{array}{llll}
s^{3} & s^{2} & s & 1
\end{array}\right] \frac{1}{8 l_{s}^{3}}\left[\begin{array}{cccc}
16 & 8 l_{s} & -16 & 8 l_{s} \\
0 & -4 l_{s}^{2} & 0 & 4 l_{s}^{2} \\
-12 l_{s}^{2} & -2 l_{s}^{3} & 12 l_{s}^{2} & -2 l_{s}^{3} \\
4 l_{s}^{3} & l_{s}^{4} & 4 l_{s}^{3} & -l_{s}^{4}
\end{array}\right]^{w_{1}} \begin{array}{l}
\phi_{1} \\
w_{2} \\
\phi_{2}
\end{array}\right] \\
& =\left[\begin{array}{l}
{\left[s_{H e 3}\right.}
\end{array}\right]\left[S h_{H e 3 c c}\right]^{i}\left[w_{h}\right] .
\end{aligned}
$$

The GBT modal segment matrices $\left[v_{h}\right]_{\text {seg }}$ and $\left[w_{h}\right]_{\text {seg }}$ follow the same procedure of longitudinal displacement.

Concerning the rotational degrees of freedom, one can directly set up the coupling of transversal rotation, $v$, as shown in Fig. 8a, between the shell elements and the generic-segmented cross-section based on the GBT definition of longitudinal rotation:

$v(x, s)=\sum_{i=1}^{n}{ }^{i} w(s)^{i} V_{, x}(x)$

Thus, the transversal rotation coupling can be expressed by the multi-freedom constraint transformation matrix, $\left[T_{c}\right]_{w}$, and GBT's degrees of freedom of generalized rotation, $\left[V_{, x}\right]_{\mathrm{GBT}}$, which are given in Eqs. 6 and 12, respectively:

$[v]_{\text {shell }}=\left[T_{c}\right]_{w}\left[V_{, x}\right]_{\mathrm{GBT}}$.

In the more generic case, in which the shell nodes lie at an arbitrary point in the GBT segment, the coupling expression is given by the following: $\left[\begin{array}{c}v_{1} \\ v_{2} \\ v_{3} \\ v_{4} \\ \vdots \\ v_{n}\end{array}\right]_{\text {shell }}=[T S 3]_{\text {seg }}\left[w_{h}\right]_{\text {seg }}\left[\begin{array}{c}{ }^{2} V_{, x} \\ { }^{3} V_{, x} \\ { }^{4} V_{, x} \\ \vdots \\ { }^{n} V_{, x}\end{array}\right]_{\mathrm{GBT}}$.

Following a similar procedure, one obtains the coupling of longitudinal rotation, $\varphi$, which is based on GBT's definition of longitudinal rotation:

$\varphi(x, s)=\sum_{i=1}^{n}{ }^{i} w_{, s}(s){ }^{i} V(x)$

If GBT's discretization and shell nodes share the same position, no interpolation of the GBT segment is necessary. Thereby, one finds the following:

$$
[\varphi]_{\text {shell }}=\left[T_{c}\right]_{\varphi}[V]_{\mathrm{GBT}} ;
$$$$
\left[\begin{array}{c}
\varphi_{1} \\
\varphi_{2} \\
\varphi_{3} \\
\varphi_{4} \\
\vdots \\
\varphi_{n}
\end{array}\right]_{\text {shell }}=\left[\begin{array}{ccccc}
{ }^{2} \varphi_{1} & { }^{3} \varphi_{1} & { }^{4} \varphi_{1} & \ldots & { }^{n} \varphi_{1} \\
{ }^{2} \varphi_{2} & { }^{3} \varphi_{2} & { }^{4} \varphi_{2} & \ldots & { }^{n} \varphi_{2} \\
{ }^{2} \varphi_{3} & { }^{3} \varphi_{3} & { }^{4} \varphi_{3} & \ldots & { }^{n} \varphi_{3} \\
{ }^{2} \varphi_{4} & { }^{3} \varphi_{4} & { }^{4} \varphi_{4} & \ldots & { }^{n} \varphi_{4} \\
\vdots & \vdots & \vdots & \ddots & \vdots \\
{ }^{2} \varphi_{n} & { }^{3} \varphi_{n} & { }^{4} \varphi_{n} & \cdots & { }^{n} \varphi_{n}
\end{array}\right]\left[\begin{array}{c}
{ }^{2} V \\
{ }^{3} V \\
{ }^{4} V \\
\vdots \\
{ }^{n} V
\end{array}\right]_{\mathrm{GBT}}
$$

Otherwise, the coupling by the interpolated values inside of a GBT segment can be expressed as follows:

$\left[\begin{array}{c}\varphi_{1} \\ \varphi_{2} \\ \varphi_{3} \\ \varphi_{4} \\ \vdots \\ \varphi_{n}\end{array}\right]_{\text {shell }}=\left[T S 3_{, s}\right]_{\text {seg }}\left[w_{h}\right]_{\text {seg }}\left[\begin{array}{c}{ }^{2} V \\ { }^{3} V \\ { }^{4} V \\ \vdots \\ { }^{n} V\end{array}\right]_{\mathrm{GBT}}$

where the subindex ${ }_{,}$indicates the first derivative of the interpolation segment matrix $[T S 3]_{\text {seg }}$.

The transversal perpendicular rotational degree of freedom, $\chi$, shown in Fig. 8a requires special remarks.

The first one is regarding the GBT's assumption of a linear variation of the longitudinal displacement in each cross-section segment. This leads to a constant transversal perpendicular rotation in each segment. Thus, if GBT's discretization and shell nodes do not share the same position, one can reach the following expression: 


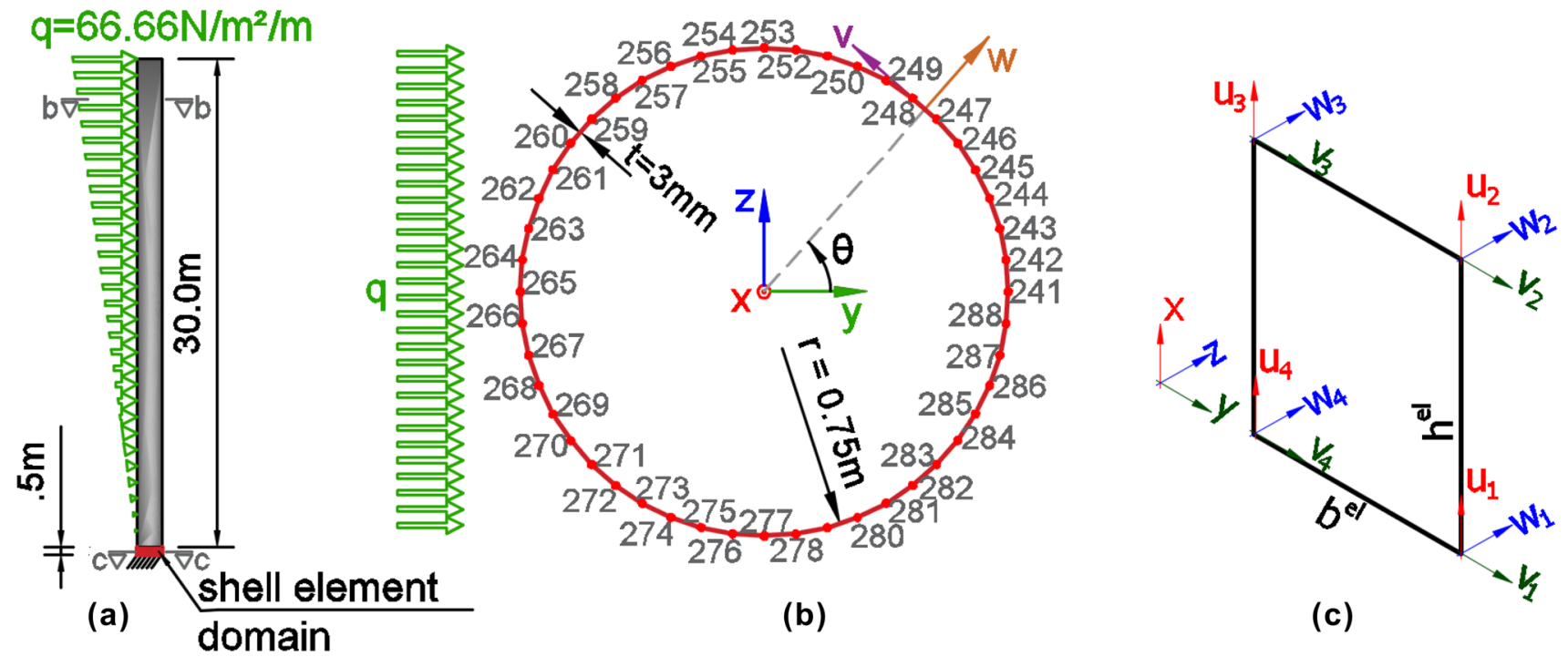

Fig. 10 Thin-walled circular hollow section under a linear projected force and a coupled shell element segment; $\mathbf{a}$ elevation; $\mathbf{b}$ cross section in GBT's domain; c cross section in shell's domain at the coupling point

$$
\left[\begin{array}{c}
\chi_{1} \\
\chi_{2} \\
\chi_{3} \\
\chi_{4} \\
\vdots \\
\chi_{n}
\end{array}\right]_{\text {shell }}=\left[T_{c}\right]_{\chi}\left[V_{, x}\right]_{\mathrm{GBT}}=\left[T S 1_{, s}\right]_{s e g}\left[u_{h}\right]_{s e g}\left[\begin{array}{c}
{ }^{1} V \\
{ }^{2} V_{, x} \\
{ }^{3} V_{, x} \\
\vdots \\
{ }^{n} V_{, x}
\end{array}\right]_{\mathrm{GBT}}
$$

However, since each segment can have a different constant value of this rotation, this leads to a discontinuity at the GBT nodes of cross-section discretization. Consequently, if GBT's discretization and shell nodes share the same position, there are ambiguous values of GBT transversal perpendicular rotation to couple.

In a simple-minded approach, this problem can be overcome using the average of the transversal perpendicular rotations of each segment that reach the node.

The second remark is about the type of shell element used. Many shell elements do not have this degree of freedom, which is named in the literature as the drilled degree of freedom. For these shell elements, there is no coupling for this transversal rotation, and all information about it vanishes.

\section{Coupling with a different global coordinate system-the hollow circular cross-section case}

It is important to observe that the approach presented up to this point considers a common coordinate system between the shell and GBT elements. Usually, this condition is found in the generic-segmented cross section. However, for a hollow circular cross section, with GBT's formulation, the coordinate systems are not the same, such as the local coordinate system presented in Fig. 10b, which is left-handed. Therefore, coupling for the hollow circular cross section requires an extra transformation for the displacements $v$ and $w$ :

$\left[\begin{array}{l}{[v]_{\text {shell }}} \\ {[w]_{\text {shell }}}\end{array}\right]_{\text {Scs }}=[T]_{\theta}\left[\begin{array}{l}{[v]_{\text {shell }}} \\ {[w]_{\text {shell }}}\end{array}\right]_{\text {GBTcs }}$

with:

$[T]_{\theta}=\left[\begin{array}{cccccc}-s\left(\theta_{1}\right) & \ldots & 0 & c\left(\theta_{1}\right) & \ldots & 0 \\ \vdots & \ddots & \vdots & \vdots & \ddots & \vdots \\ 0 & \ldots & -s\left(\theta_{n}\right) & 0 & \ldots & c\left(\theta_{n}\right) \\ c\left(\theta_{1}\right) & \ldots & 0 & s\left(\theta_{1}\right) & \ldots & 0 \\ \vdots & \ddots & \vdots & \vdots & \ddots & \vdots \\ 0 & \ldots & c\left(\theta_{n}\right) & 0 & \ldots & s\left(\theta_{n}\right)\end{array}\right]$

where $s$ and $c$ are the abbreviations of sine and cosine of angle $\theta_{n}$, which is the angle of each shell node according to GBT's local coordinate system, as shown in Fig. 10b; the

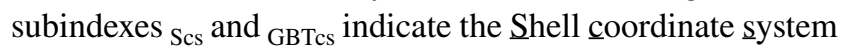
and the GBT's coordinate system.

The transversal rotations require the same treatment:

$\left[\begin{array}{l}{[v]_{\text {shell }}} \\ {[\chi]_{\text {shell }}}\end{array}\right]_{\text {Scs }}=[T]_{\theta}\left[\begin{array}{l}{[v]_{\text {shell }}} \\ {[\chi]_{\text {shell }}}\end{array}\right]_{\text {GBTcs }}$ 
Table 1 Relevant orthogonal deformation shape modes in this example

\begin{tabular}{lllll}
\hline $\mathrm{i}$ & $\mathrm{m}$ & ${ }^{i} u$ & ${ }^{i} v^{\star}$ & ${ }^{i} w$ \\
\hline $\mathrm{a}$ & 0 & 0 & 0 & 1 \\
3 & 1 & $-r \cos (\theta)$ & $-\sin (\theta)$ & $\cos (\theta)$ \\
5 & 2 & $-r \cos (2 \theta)$ & $-2 \sin (2 \theta)$ & $2^{2} \cos (2 \theta)$ \\
7 & 3 & $-r \cos (3 \theta)$ & $-3 \sin (3 \theta)$ & $3^{2} \cos (3 \theta)$ \\
11 & 5 & $-r \cos (5 \theta)$ & $-5 \sin (5 \theta)$ & $5^{2} \cos (5 \theta)$ \\
\hline
\end{tabular}

\section{Coupled stiffness matrix and external load vector}

Once the multi-freedom constraint matrices are evaluated, the next step in the mixed shell-GBT model is the setup of coupled stiffness matrix and the external load vector. This is easily achieved by the respective matrix transformations:

$[K]_{c p}=\left[T_{c}\right]_{t o t}^{T}[K]_{u c}\left[T_{c}\right]_{t o t}$

$[F]_{c p}=\left[T_{c}\right]_{t o t}^{T}[F]_{u c}$,

where the subindexes ${ }_{c p}$ and ${ }_{u c}$ indicate the coupled and uncoupled matrices, respectively. The total coupling transformation matrix, $\left[T_{c}\right]_{t o t}$, is an identity matrix of the same rank as the uncoupled stiffness matrix, which has the columns concerning the coupled shell's degrees of freedom removed. Furthermore, the rows concerning these degrees of freedom are re-populated by the values of matrices $\left[T_{c}\right]_{u}$, $\left[T_{c}\right]_{v},\left[T_{c}\right]_{w},\left[T_{c}\right]_{\varphi}$ and $\left[T_{c}\right]_{\chi}$. The next section shows the implementation of this matrix via a detailed example.

\section{Numerical example of coupling of GBT and shell elements}

As a detailed numerical example of the application of coupling between the shell and GBT elements, let us consider the thin-walled circular hollow steel cross section, as shown in Fig. 10. This cross section is applied in a vertical cantilever structure subjected to a linear projected surface load. That is, the total load applied on the structure is not a product of the surface load and the area of the surface; it is the product of the surface load and the projected area on the global coordinate direction $\mathrm{z}$. The material parameters are Young's modulus $E=205,000 \mathrm{~N} / \mathrm{mm}^{2}$, Poisson's ratio $\mu=0.3$, and shear modulus $G=78,846.2 \mathrm{~N} / \mathrm{mm}^{2}$.

\section{Setup of finite-element and coupling matrices}

The structure is $30.5 \mathrm{~m}$ high, of which the upper $30 \mathrm{~m}$ is modeled by 1 exact GBT element (Bianco et al. 2016), with
Table 2 Setup of degrees of freedom in the mixed model

\begin{tabular}{lllll}
\hline Displ. & DOF's & Support restrain & $\begin{array}{l}\text { Master } \\
\text { DOF }\end{array}$ & $\begin{array}{l}\text { Slave } \\
\text { DOF }\end{array}$ \\
\hline u & 1 to 288 & $1-48$ & - & $241-288$ \\
v & $289-576$ & $289-337$ & - & $529-576$ \\
w & $577-864$ & $577-625$ & - & $817-864$ \\
${ }^{3} V$ & $865 ; 867 ; 869$ & - & 865 & - \\
${ }^{3} V_{, x}$ & $866 ; 868 ; 870$ & - & 866 & - \\
${ }^{5} V$ & $871 ; 873 ; 875$ & - & 871 & - \\
${ }^{5} V_{, x}$ & $872 ; 874 ; 876$ & - & 872 & - \\
${ }^{7} V$ & $877 ; 879 ; 881$ & - & 877 & - \\
${ }^{7} V_{, x}$ & $878 ; 880 ; 882$ & - & 878 & - \\
${ }^{11} V$ & $883 ; 885 ; 887$ & - & 883 & - \\
${ }^{11} V_{, x}$ & $884 ; 886 ; 888$ & - & 884 & - \\
${ }^{15} V$ & $889 ; 891 ; 893$ & - & 889 & - \\
${ }^{15} V_{, x}$ & $890 ; 892 ; 894$ & - & 890 & - \\
${ }^{a} V$ & $895 ; 897 ; 899$ & - & 895 & - \\
${ }^{a} V_{, x}$ & $896 ; 898 ; 900$ & - & 896 & - \\
\hline
\end{tabular}

3 nodes per element, 2 degrees of freedom per node, and 5 deformation modes: 1 for radial deformation $(\mathrm{i}=\mathrm{a}) ; 1$ for bending ( $(=3)$; and 3 for ovalization ( $i=5,7$, and 11). The respective transversal and longitudinal displacements of these modes are provided in Table. 1. These lead to a total of 30 degrees of freedom.

The lower half meter is discretized by shell elements, including 48 node elements in the transversal direction and five layers of elements in the longitudinal direction. Thus, the shell part of the model has 288 nodes and 240 shell elements. Consequently, all the elements have the same proportion and shape. Moreover, they are limited to the well-known membrane behavior (Bathe 1996), which leads to 3 degrees of freedom per node. Therefore, a total of 864 degrees of freedom is obtained. From this total, 48 nodes (144 degrees of freedom at the base) are restrained to the displacement. The same amount will be restrained at the top cross section of this prolongation by the multi-freedom constraint matrix. The final mixed model has 576 free degrees of freedom from the shell part.

The transformation from the local (shown in Fig. 10c) to global coordinate system of each shell element and the assembly of the global stiffness matrix follows the wellknown approach of FEM (Bathe 1996). To this global stiffness obtained from the shell elements, the GBT stiffness matrices are added, which, in this step, are still uncoupled among each other, as well as among the membrane's degrees of freedom. Table 2 resumes the setup of the degrees of freedom. 
Table 3 Solution of GBT's degrees of freedom, in $\mathrm{mm}$

\begin{tabular}{lrcrrrr}
\hline Mode & $V\left(x=-\frac{L}{2}\right)$ & $V_{, x}\left(x=-\frac{L}{2}\right)$ & \multicolumn{1}{l}{$V(x=0)$} & $V_{, x}(x=0)$ & $V\left(x=\frac{L}{2}\right)$ & $V_{, x}\left(x=\frac{L}{2}\right)$ \\
\hline 3 & $1.793 \mathrm{E}-1$ & $5.535 \mathrm{E}-4$ & $2.901 \mathrm{E}+2$ & $1.298 \mathrm{E}-2$ & $1.024 \mathrm{E}+2$ & $1.116 \mathrm{E}-2$ \\
5 & $-1.302 \mathrm{E}-2$ & $-4.488 \mathrm{E}-5$ & $-1.325 \mathrm{E}+1$ & $-4.788 \mathrm{E}-4$ & $-5.867 \mathrm{E}+0$ & $-5.130 \mathrm{E}-4$ \\
7 & $6.048 \mathrm{E}-4$ & $2.088 \mathrm{E}-6$ & $2.753 \mathrm{E}-1$ & $9.021 \mathrm{E}-6$ & $1.383 \mathrm{E}-1$ & $9.172 \mathrm{E}-6$ \\
11 & $-6.473 \mathrm{E}-6$ & $-2.351 \mathrm{v} 8$ & $-1.573 \mathrm{v} 3$ & $-4.965 \mathrm{E}-8$ & $-7.885 \mathrm{E}-4$ & $-5.257 \mathrm{E}-8$ \\
Axial & $7.619 \mathrm{E}-15$ & $-1.524 \mathrm{E}-8$ & $-4.573 \mathrm{E}-4$ & $-1.524 \mathrm{E}-8$ & $-2.287 \mathrm{E}-4$ & $-1.524 \mathrm{E}-8$ \\
\hline
\end{tabular}

\section{Coupling of longitudinal displacement-u}

To couple the longitudinal displacement, $u$, one must evaluate the multi-freedom constraint transformation matrix, $\left[T_{c}\right]_{u}$, for each slave degree of freedom with respect to each GBT mode. To do so, it is necessary to compute the modal longitudinal displacement of each slave node, given in Table 1 , with the respective node angle $\theta$, where the $\Delta \theta$ between the nodes is $7.5^{\circ}$, as presented in Fig. 10b. These results are introduced into Eq. 6, leading to the following:

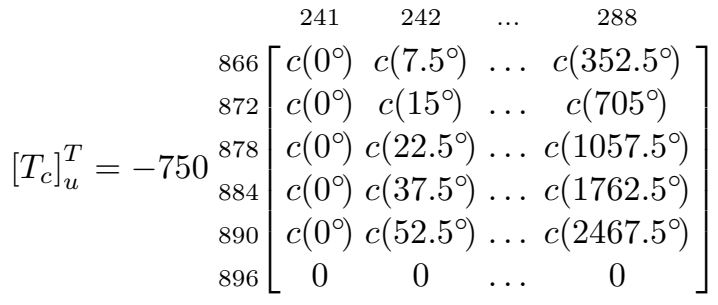

On the left and on the top, the row and column indexes indicate the master and slave degrees of freedom, respectively. Since the longitudinal displacement of GBT is consistent with the global coordinate system of the membranes, no extratransformation is required for these degrees of freedom.

\section{Coupling of transversal displacements $-v$ and $w$}

Similar to the coupling of longitudinal displacement, the transversal coupling is obtained by the multi-freedom constraint transformation matrices $\left[T_{c}\right]_{v}$ and $\left[T_{c}\right]_{w}$ (Eqs. 11 and 12, respectively), which are based on the expression of Table 1 . Therefore, one can find the following:

$$
\left.\left[T_{c}\right]_{v}^{T}=-1 \begin{array}{ccccc}
529 & 530 & \ldots & 576 \\
865 & 871 \\
883 & s\left(0^{\circ}\right) & s\left(7.5^{\circ}\right) & \ldots & s\left(352.5^{\circ}\right) \\
2 s\left(0^{\circ}\right) & 2 s\left(15^{\circ}\right) & \ldots & 2 s\left(705^{\circ}\right) \\
8 s\left(0^{\circ}\right) & 3 s\left(22.5^{\circ}\right) & \ldots & 3 s\left(1057.5^{\circ}\right) \\
889 & 5 s\left(0^{\circ}\right) & 5 s\left(37.5^{\circ}\right) & \ldots & 5 s\left(1762.5^{\circ}\right) \\
8 s\left(0^{\circ}\right) & 7 s\left(52.5^{\circ}\right) & \ldots & 7 s\left(2467.5^{\circ}\right) \\
0 & 0 & \ldots & 0
\end{array}\right]
$$

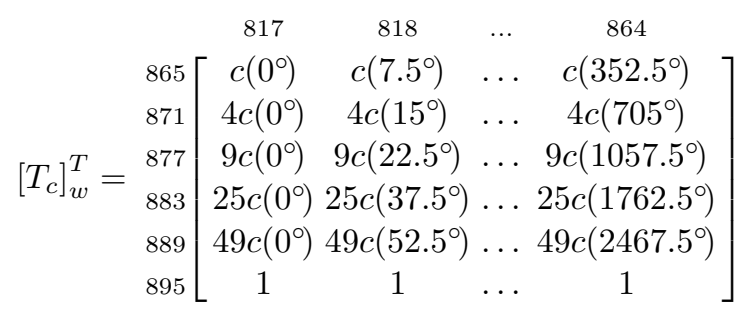

Different from the longitudinal displacement, the GBT transversal ones are not in the global membrane's coordinate system. Thereby, the transformation given in Eq. 25 is required, which leads to the following:

$$
\left[T_{c}\right]_{v w}=
$$

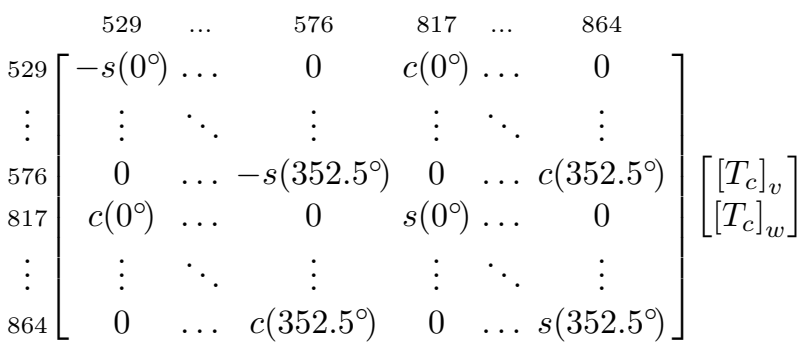

\section{Finite-element solution}

Once the multi-freedom constraint transformation matrices are evaluated for each displacement direction, a total form of these matrices, $\left[T_{c}\right]_{t o t}$, can be built up.

As mentioned before, initially, an identity matrix, $I$, is defined. Its dimensions have the same number of no-support degrees of freedom, $756 \times 756$. From this matrix, all columns corresponding to slave degrees of freedom are removed, which leads to the dimension $756 \times 612$. Thereafter, each component of the rows that concern the slave degrees of freedom is replaced by the respective term from the matrices $\left[T_{c}\right]_{u}$ and $\left[T_{c}\right]_{v w^{*}}$.

With the definition of the total multi-freedom constraint transformation matrix, one can achieve the coupled free 


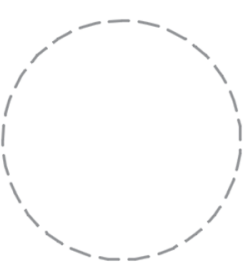

axial mode

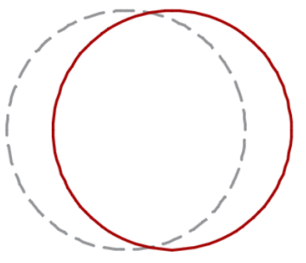

mode 3

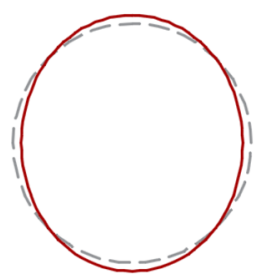

mode 5

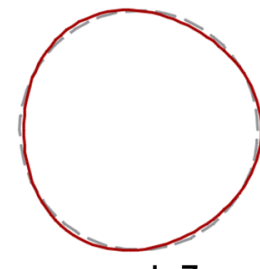

mode 7 (scaled by 10 )

-- cross-section middle wall $=$ GBT solution $\quad x \times \times$ shell $63 \Delta \Delta \Delta$ shell 93

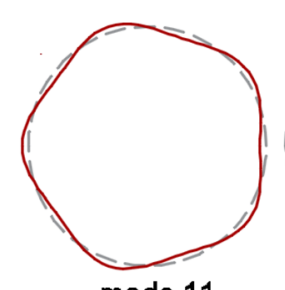

mode 11

(scaled by $10^{\wedge} 3$ )

प्व shell 181

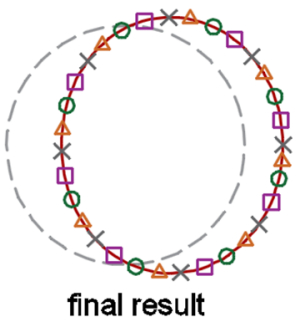

๑ө shell 281

Fig. 11 Results of top cross section: transversal displacement of mixed shell-GBT element model. The GBT solution is obtained by the summation at the top point of all the modal deformation factors. The solutions achieved from the fully shell elements models are also presented

Table 4 Mean differences, in $\%$, and standard deviation (SD) of the displacement field between GBT and shell element models at the top point

\begin{tabular}{|c|c|c|c|c|c|c|}
\hline \multirow[t]{2}{*}{ Element } & \multicolumn{2}{|l|}{$u$} & \multicolumn{2}{|l|}{$v$} & \multicolumn{2}{|l|}{$w$} \\
\hline & Diff. (\%) & $\mathrm{SD}(\%)$ & Diff. (\%) & $\mathrm{SD}(\%)$ & Diff. (\%) & $\mathrm{SD}(\%)$ \\
\hline Shell-63 & 0.16 & 0.34 & 0.7 & 0.12 & 0.6 & 0.3 \\
\hline Shell-93 & 0.08 & 0.24 & 0.62 & 0.07 & 0.55 & 0.18 \\
\hline Shell-181 & 0.17 & 0.17 & 0.93 & 0.94 & 0.84 & 0.86 \\
\hline Shell-281 & 0.62 & 0.07 & 0.54 & 0.19 & 0 & 0 \\
\hline
\end{tabular}

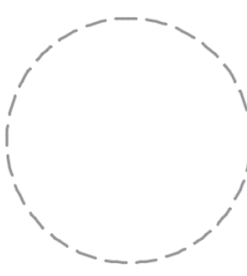

axial mode
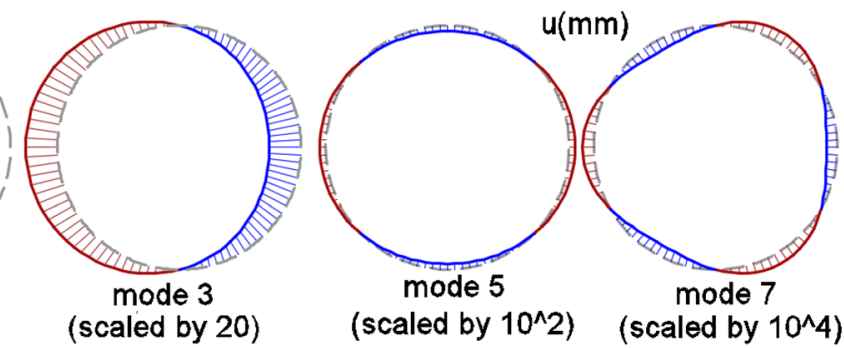

(scaled by $10^{\wedge} 4$ )

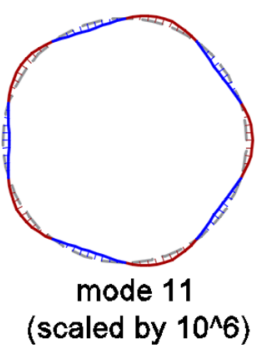

(scaled by $10^{\wedge} 6$ )

마 shell 181

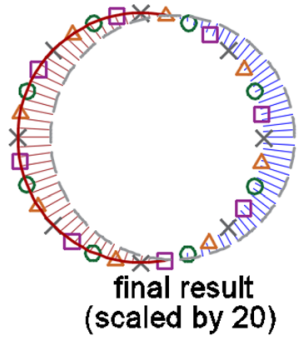

ooo shell 281

Fig. 12 Comparison of results among mixed shell-GBT and fully shell models at the top cross section: longitudinal displacement

stiffness matrix $[K]_{c p}$ by Eq. 26, in which the dimensions are $612 \times 612$.

Since, in this example, there is no external load applied to the slave degrees of freedom, the external load vector can be directly assembled without the related rows. Alternatively, one can apply Eq. 27. The same external load vector is reached.

Finally, the numerical values of the degrees of freedom are achieved by a standard solver, such as the Cholesky decomposition, which is used here. Table 3 presents the numerical solution for GBT elements:

One important remark concerns the indirect coupling among the GBT's degrees of freedom of orthogonal modes. Unlike the GBT's linear analysis, where each model is solved completely isolated from the others, in the mixed model, this is no longer possible. The multi-freedom constraint transformation matrices build this indirect coupling, which requires solving a relative large linear system.

\section{Analysis of displacement field}

To perform an evaluation of the results obtained in the mixed model above, four full-shell element models are used as a control parameter, obtained from commercial ANSYS ${ }^{\circledR}$ software. The difference among these models is the type of interpolation function applied: linear (shell-63 and shell181) or quadratic (shell-93 and shell-281). These elements are based on one of the following kinematic hypotheses: Kirchhoff-Love (shell-63 and shell-93) and Mindlin-Reissner (shell-181 and shell-281). All models have the same discretization, which has 100 elements in the cross section and 610 segments in the longitudinal direction. In total, each model has 61,000 elements, which leads to 366,600 and $1,099,200$ degrees of freedom for the linear and quadratic element models, respectively. 

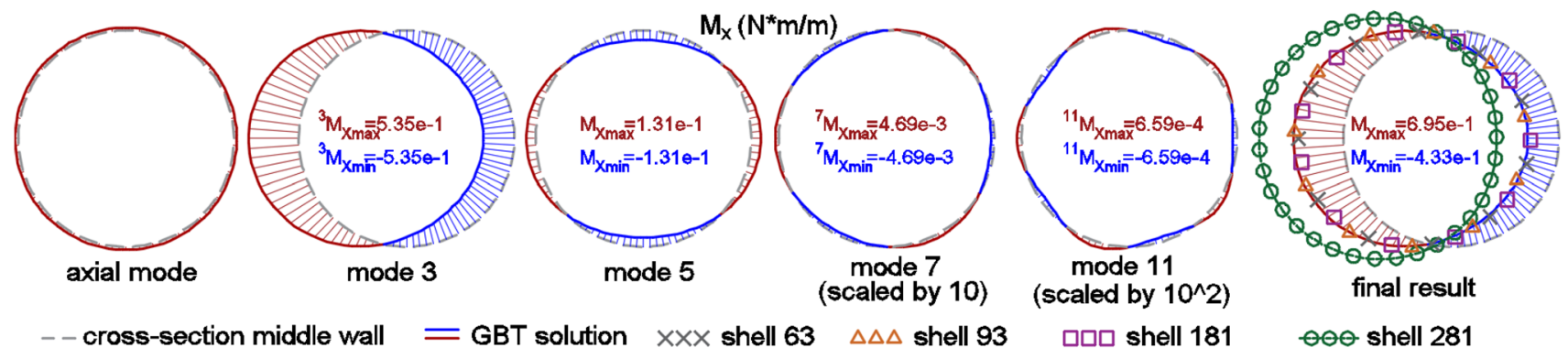

प्र shell 181

๑॰ shell 281

Fig. 13 Comparison of results among mixed shell-GBT and fully shell models at point $x=525 \mathrm{~mm}$ : longitudinal bending moment, $M_{x}$

Table 5 Longitudinal bending moment, $M_{x}$, at $x=525 \mathrm{~mm}$ : mean differences (in the table's upper right side) and their standard deviation (in the table's lower left side) among all models

\begin{tabular}{llllll}
\hline$M_{x}$ & GBT (\%) & Shell-63 (\%) & Shell-93 (\%) & Shell-181 (\%) & Shell-281 (\%) \\
\hline GBT & - & -9.22 & -0.59 & -9.45 & 79.57 \\
Shell-63 & 2.09 & - & -8.57 & 0.25 & 95.65 \\
Shell-93 & 4.64 & 2.3 & - & -8.8 & 77.55 \\
Shell-181 & 2.07 & 0.02 & 2.31 & - & 95.38 \\
Shell-281 & 19.48 & 21.33 & 22.18 & 28.31 & - \\
\hline
\end{tabular}

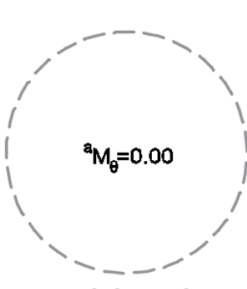

axial mode

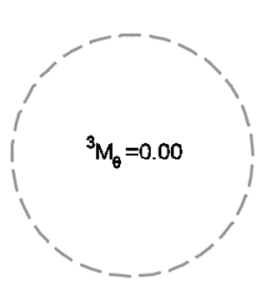

mode 3

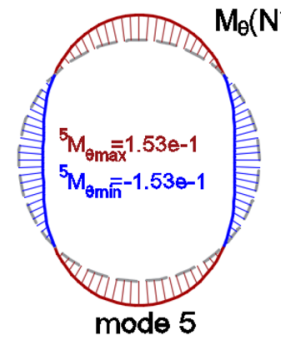

- - cross-section middle wall $=$ GBT solution $x \times x$ shell $63 \Delta \Delta \Delta$ shell 93

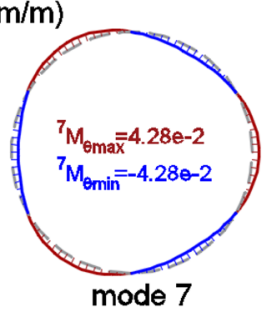

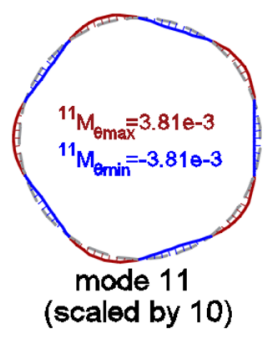

प्र shell 181

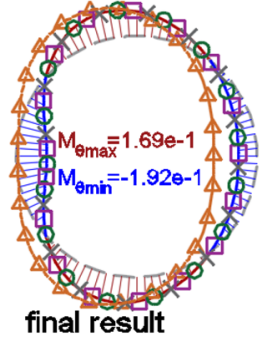

shell 281

Fig. 14 Comparison of results among mixed shell-GBT and fully shell models at point $x=525 \mathrm{~mm}$ : transversal bending moment, $M_{\theta}$

Table 6 Transversal bending moment, $M_{\theta}$, at $x=525 \mathrm{~mm}$ : mean differences (in the table's upper right side) and their standard deviation (in the table's lower left side) among all models

\begin{tabular}{llllll}
\hline$M_{\theta}$ & GBT $(\%)$ & Shell-63 $(\%)$ & Shell-93 $(\%)$ & Shell-181 $(\%)$ & Shell-281 (\%) \\
\hline GBT & - & 0.52 & 41.17 & 0.59 & 0.61 \\
Shell-63 & 0.48 & - & -77.16 & -0.06 & 0.09 \\
Shell-93 & 192.69 & 129.06 & - & -77.22 & -77.04 \\
Shell-181 & 0.89 & 0.49 & 131.32 & - & 0.03 \\
Shell-281 & 0.29 & 0.3 & 128.97 & 0.78 & - \\
\hline
\end{tabular}

Figure 11 plots the comparison among all the models for the transversal displacement at the top cross section. The modal displacement field of GBT is plotted, as well.

Here, the results of transversal displacements obtained from different fully shell element models are almost the same. As shown in Table 4, the highest mean difference is $0.93 \%$, which occurs between GBT and the model with shell type 281 . In addition, the standard deviation is on the same order: $0.94 \%$. The best agreement of results occurs between GBT and the shell-93 model, which have a mean difference and a standard deviation of $0.62 \%$ and $0.07 \%$, respectively.

The diagrams of longitudinal displacements are plotted in Fig. 12, as well. Similar to transversal displacements, the differences among the models are almost imperceptible. The highest value of the mean difference is $0.62 \%$, which is between GBT and shell element type 281. 


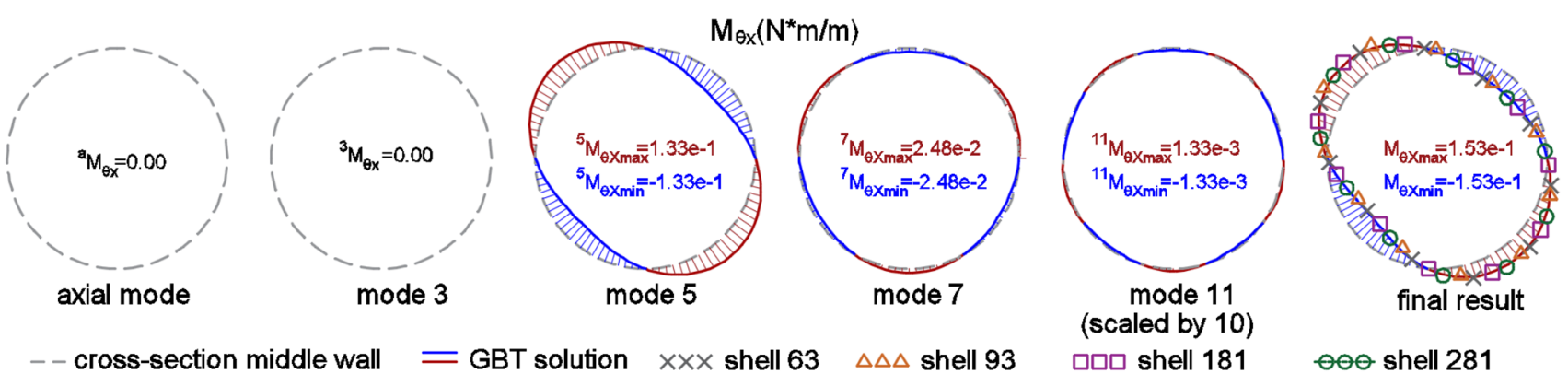

Fig. 15 Comparison of results among mixed shell-GBT and fully shell models at point $x=525 \mathrm{~mm}$ : twist moment, $M_{\theta x}$

Table 7 Twist bending moment, $M_{\theta x}$, at $x=525 \mathrm{~mm}$ : mean differences (in the table's upper right side) and their standard deviation (in table's lower left side) among all models

\begin{tabular}{llllll}
\hline$M_{\theta x}$ & GBT $(\%)$ & Shell-63 $(\%)$ & Shell-93 $(\%)$ & Shell-181 (\%) & Shell-281 (\%) \\
\hline GBT & - & 6.7 & 6.77 & 6.08 & 7 \\
Shell-63 & 5.1 & - & 3.67 & 1.07 & 0.22 \\
Shell-93 & 18.41 & 19.34 & - & 1.1 & 3.97 \\
Shell-181 & 6.05 & 8.2 & 11 & - & 1.27 \\
Shell-281 & 6.35 & 1.11 & 20.39 & 9.12 & - \\
\hline
\end{tabular}

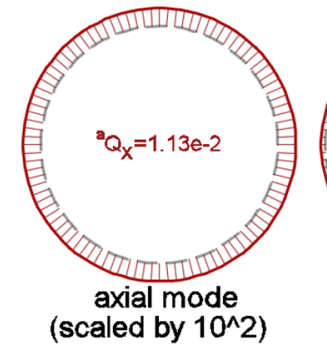

(scaled by 10)

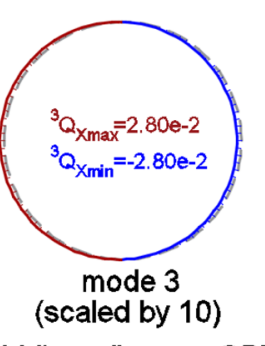

GBT solution
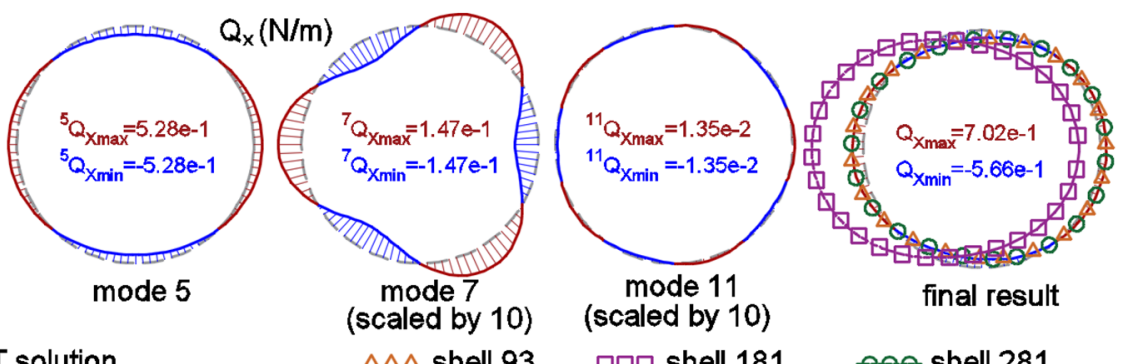

$\Delta \Delta \Delta$ shell 93

प्व shell 181

๑o shell 281

Fig. 16 Comparison of results among mixed shell-GBT and fully shell models at point $x=525 \mathrm{~mm}$ : longitudinal shear force, $Q_{x}$

Table 8 Longitudinal plate's shear, $Q_{x}$, at $x=525 \mathrm{~mm}$ : mean differences (in the table's upper right side) and their standard deviation (in the table's lower left side) among all models

\begin{tabular}{lllll}
\hline$Q_{x}$ & GBT (\%) & Shell-93 (\%) & Shell-181 (\%) & Shell-281 (\%) \\
\hline GBT & - & 7.29 & 55.43 & 7.21 \\
Shell-93 & 17.05 & - & -22 & 1.34 \\
Shell-181 & 401.58 & 544 & - & -69.95 \\
Shell-281 & 8.59 & 10.1 & 70.59 & - \\
\hline
\end{tabular}

\section{Analysis of stress field}

The comparisons among the mixed GBT-shell and the fullshell element models are placed at the longitudinal position of $x=525 \mathrm{~mm}$. This point is in the GBT domain in the mixed model and at the center of the first elements after the coupling cross section in the full-shell element models. For this cross section, one can obtain the following internal bending moments (Figs. 13, 14, 15, 16).

Table 5 shows that the GBT result approaches the solution of shell-93. In addition, it indicates that there is no consensus among the different shell element models regarding the values of the plate's bending moment. Shell-281 has a clear deviation from the result of the other models.

Although shell-93 gives the closest result to GBT in terms of the longitudinal bending moment, it provides the farthest one concerning the transversal bending moment, as shown in Table 6.

Concerning the twist bending moment, $M_{\theta x}$, GBT's results diverge from the shell-93 ones and converge to those of the other models. Similar to longitudinal bending moment, there is an increase in the mean difference among GBT and all shell models, simultaneously with a decrease in the standard deviation (Tables 7, 8, 9, 10, 11, 12). 


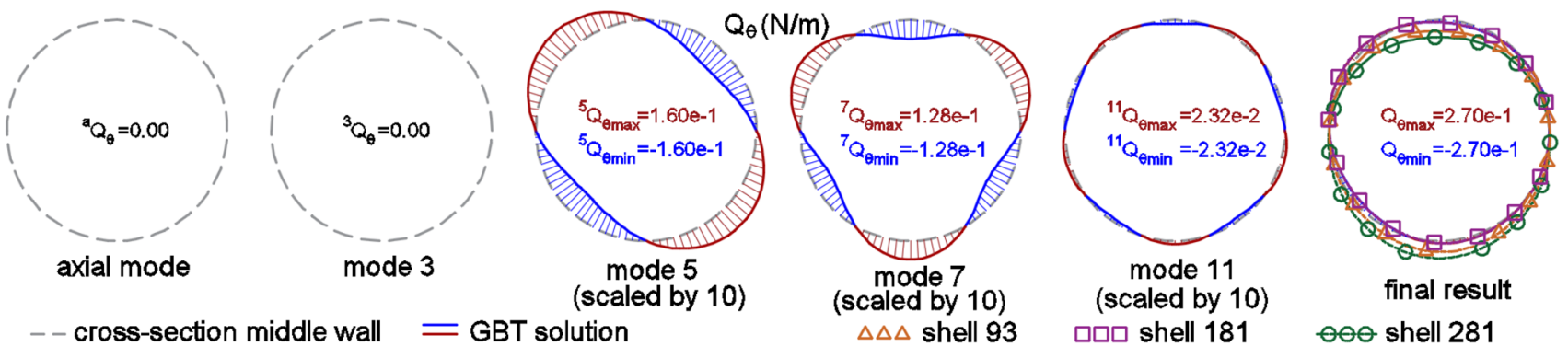

Fig. 17 Comparison of results among mixed shell-GBT and fully shell models at point $x=525 \mathrm{~mm}$ : transversal shear force, $Q_{\theta}$

Table 9 Transversal plate's shear, $Q_{\theta}$, at $x=525 \mathrm{~mm}$ : mean differences (in the table's upper right side) and their standard deviation (in the table's lower left side) among all models

\begin{tabular}{lllll}
\hline$Q_{\theta}$ & GBT (\%) & Shell-93 (\%) & Shell-181 (\%) & Shell-281 (\%) \\
\hline GBT & - & 73.27 & 1.91 & 23.34 \\
Shell-93 & 362.26 & - & -151.6 & 87.48 \\
Shell-181 & 6.67 & 87.84 & - & 247.31 \\
Shell-281 & 496.43 & 51.87 & 852.02 & - \\
\hline
\end{tabular}

Following the same representation, the internal shear forces can be compared to the results from FEM based on shell elements, which indicates that GBT's longitudinal shear force, $Q_{x}$, approaches the results found for shell281. Shell-181 exhibits a strong difference for this internal force. As presented in Fig. 17, the results of this shell model not only show a quantitative difference, but also show a considerable qualitative one.

On the other hand, in the analysis of transversal shear force, shell-181 presents a lower difference from the GBT results, with a mean difference of $1.91 \%$ and a standard deviation of $6.67 \%$.

The lack of consensus on the plate's internal forces does not occur in the case of longitudinal and shear membrane forces. The proposed GBT-shell coupling model and all shell models lead almost to the same solution. Each internal force is compared to the result of the shell finiteelement models in the cross section at point $x=525 \mathrm{~mm}$ as well:

However, the approximation of the cross-section analysis to the support conditions is reflected in the membrane's result in the transversal direction, $N_{\theta}$ : Shell-181 presents higher results than any other model. Moreover, there is no

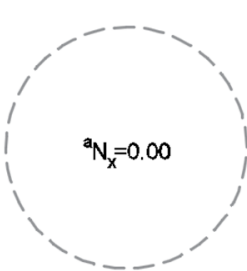

axial mode

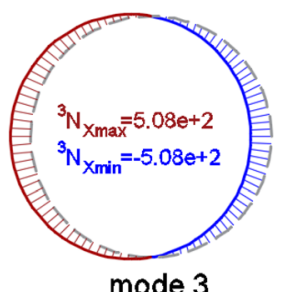

mode 3

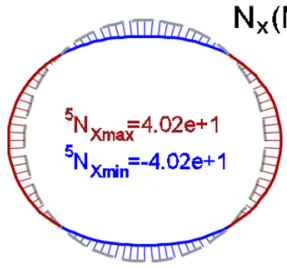

mode 5 (scaled by 10)

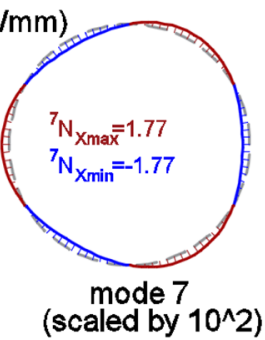

(scaled by $10^{\wedge} 2$ )

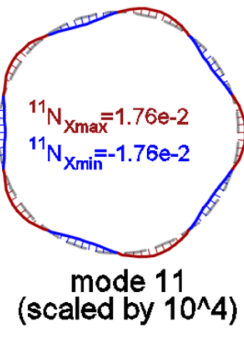

마 shell 181

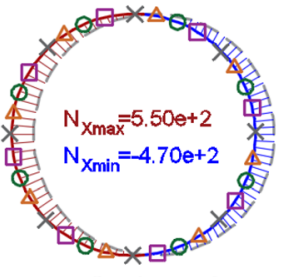

final result

-- cross-section middle wall $=$ GBT solution $\quad x \times \times$ shell $63 \Delta \Delta \Delta$ shell 93

๑o shell 281

Fig. 18 Comparison of results among mixed shell-GBT and fully shell models at point $x=525$ mm: longitudinal membrane force, $N_{x}$

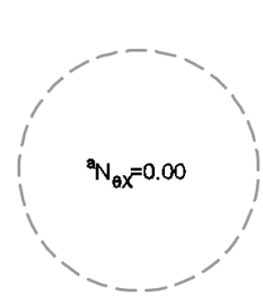

axial mode

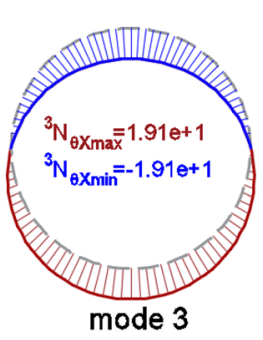

mode 3

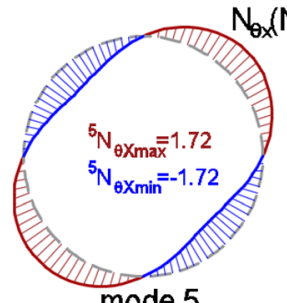

mode 5

(scaled by 10 )

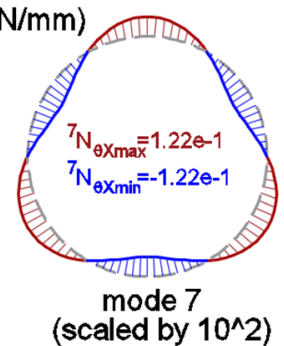

(scaled by $10^{\wedge} 2$ )

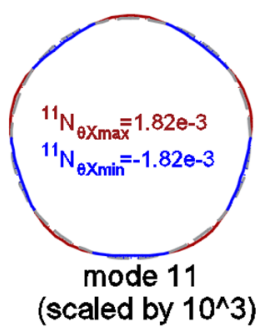

$\square \square \square$ shell 181

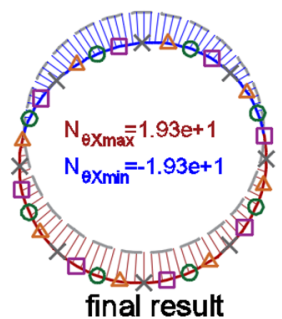

ove shell 281

Fig. 19 Comparison of results among mixed shell-GBT and fully shell models at point $x=525$ mm: shear membrane force, $N_{\theta x}$ 
Table 10 Longitudinal membrane force, $N_{x}$, at $x=525 \mathrm{~mm}$ : mean differences (in the table's upper right side) and their standard deviation (in the table's lower left side) among all models

\begin{tabular}{llllll}
\hline$N_{x}$ & GBT $(\%)$ & Shell-63 $(\%)$ & Shell-93 $(\%)$ & Shell-181 (\%) & Shell-281 (\%) \\
\hline GBT & - & 0.02 & -0.03 & 0.05 & -0.03 \\
Shell-63 & 0.17 & - & 0.05 & -0.03 & -0.05 \\
Shell-93 & 0.13 & 0.04 & - & 0.08 & 0.01 \\
Shell-181 & 0.19 & 0.02 & 0.06 & - & -0.08 \\
Shell-281 & 0.13 & 0.04 & 0 & 0.06 & - \\
\hline
\end{tabular}

Table 11 Shear membrane force, $N_{\theta x}$, at $x=525 \mathrm{~mm}$ : mean differences (in the table's upper right side) and their standard deviation (in the table's lower left side) among all models

\begin{tabular}{llllll}
\hline$N_{\theta x}$ & GBT $(\%)$ & Shell-63 $(\%)$ & Shell-93 $(\%)$ & Shell-181 $(\%)$ & Shell-281 $(\%)$ \\
\hline GBT & - & 0.02 & -0.01 & 0.02 & -0.01 \\
Shell-63 & 0.15 & - & 0.03 & 0.01 & -0.03 \\
Shell-93 & 0.12 & 0.03 & - & 0.03 & 0 \\
Shell-181 & 0.17 & 0.02 & 0.05 & - & -0.03 \\
Shell-281 & 0.13 & 0.02 & 0.01 & 0.04 & - \\
\hline
\end{tabular}

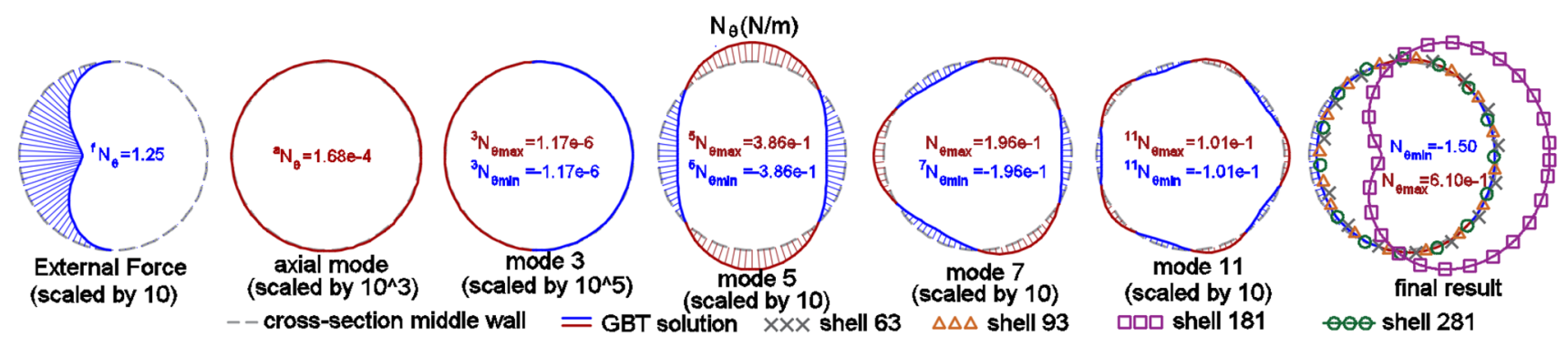

Fig. 20 Comparison of results among mixed shell-GBT and fully shell models at point $x=525 \mathrm{~mm}$ : transversal membrane force, $N_{\theta}$

Table 12 Membrane tangential force, $N_{\theta}$, at $x=525 \mathrm{~mm}$ : mean differences (in the table's upper right side) and their standard deviation (in the table's lower left side) among all models

\begin{tabular}{llllll}
\hline$N_{\theta}$ & GBT $(\%)$ & Shell-63 $(\%)$ & Shell-93 $(\%)$ & Shell-181 $(\%)$ & Shell-281 $(\%)$ \\
\hline GBT & - & 39.35 & 22.54 & 862.3 & 35.73 \\
Shell-63 & 137.35 & - & 14.87 & -82.61 & -82.2 \\
Shell-93 & 33.48 & 115.82 & - & 320.36 & 26.09 \\
Shell-181 & 3.25 & 0.87 & 2041.77 & - & -86.47 \\
Shell-281 & 36.05 & 110.91 & 64.73 & 0.79 & - \\
\hline
\end{tabular}

consensus among all models on the values of this internal force. Nevertheless, one can note that the values presented in Fig. 20 are really small compared to the values of the other membrane forces given in Figs. 18 and 19 (Fig. 20).

\section{Conclusion}

This study proposes a mixed shell-GBT model for the analysis of warping and of distortion transmission.

The key point in the proposed mixed model is the development of a novel coupling approach between shell and GBT elements, which is based on Master-Slave method.
Furthermore, the proposed coupling approach sets the GBT degrees of freedom as master ones. Thus, the coupling equations are developed from the modal displacements of GBT.

Two consequences are derived from these coupling equations: (1) the coupling among GBT modes is already in the linear analysis; (2) the displacement and stress field of shell elements, in the neighborhood of the connection, are limited to GBT's assumptions.

Despite this limitation in shell elements, the numerical example shows a high convergence in the displacement field among the proposed mixed model and different types of full-shell element models: (1) linear or 
quadratic interpolation function; (2) Kirchhoff-Love or Mindlin-Reissner hypothesis.

Concerning the stress fields, there exist imperceptible differences for the internal forces $N_{x}$ and $N_{\theta x}$. The same evaluation can be built for the plate's bending moments $M_{x}, M_{\theta}$ and $M_{\theta x}$ and longitudinal shear force $Q_{x}$. However, for these internal forces, one model of fully shell elements leads to divergent results. The GBT results are approximately the same as the other models' results.

The transversal shear force and the transversal membrane force, $Q_{\theta}$ and $N_{\theta}$, respectively, cannot be concluded based on the perturbation of the coupling procedure, as all models have no consensus on the final results. Nevertheless, these internal have a residual participation in the total stress field of thin-walled beams.

Acknowledgements This work was carried out with support of $\mathrm{CNPq}$ (Conselho Nacional de Desenvolvimento Cientfico e TecnolgicoNational Council for Scientific and Technological Development)-Brazil and Horizon 2020 MSCA-RISE-2015 project No. 691213 entitled Exchange Risk".

Open Access This article is distributed under the terms of the Creative Commons Attribution 4.0 International License (http://creativeco mmons.org/licenses/by/4.0/), which permits unrestricted use, distribution, and reproduction in any medium, provided you give appropriate credit to the original author(s) and the source, provide a link to the Creative Commons license, and indicate if changes were made.

\section{References}

Basaglia C, Camotim D, Silvestre N (2008) Global buckling analysis of plane and space thin-walled frames in the context of gbt. Thin Walled Struct 46:79-101

Basaglia C, Camotim D, Silvestre N (2012) Torsion warping transmission at thin-walled frame joints: kinematics, modelling and structural response. J Constr Steel Res 69:39-53

Bathe K (1996) Finite element procedures. Prentice-Hall, Upper Saddle River

Bianco MJ, Könke C, Habtemariam A et al (2018) Exact finite element formulation in generalized beam theory. Int J Adv Struct Eng 10:295-323. https://doi.org/10.1007/s40091-018-0199-8
Davies JM, Leach P, Heinz D (1994) Second-order generalised beam theory. J Constr Steel Res 31:221-241

Fujitani Y, Fuji D (1998) A structural analysis method of framed structures with thin-walled open section members based on the bending-torsional theory of beams. In: Idelsohn S, Oñate E, Dvorkin E (eds) Computational mechanics - new trends and applications, vol 61. CIMNE, Barcelona, Spain, pp 749-763

Jelenić G, Crisfield MA (1996) Non-linear master-slave relationships for joints in 3-d beams with large rotations. Comput Methods Appl Mech Eng 135:211-228

Munoz J, Jelenić G, Crisfield MA (2003) Master-slave approach for the modelling of joints with dependent degrees of freedom in flexible mechanisms. Commun Numer Methods Eng 19:689-702

Kindmann R, Kraus M (2011) Steel structures design using FEM. Ernst adn Sohn, Berlin

de Miranda S, Gutiérrez A, Miletta R, Ubertini F (2013) A generalized beam theory with shear deformation. Thin Walled Struct 67:88-100

Schardt R (1989) Verllgemeinerte Technische Biegetheorie. Springer, New York

Schardt R (1994) Generalized beam theory-an adequate method of coupled stability problems. Thin Walled Struct 19:161-180

Schardt R (1994) Lateral torsional and distortional buckling of channeland hat-sections. J Constr Steel Res 31:243-265

Silvestre N, Camotim D (2003) Influence of shear deformation on the local and global buckling behaviour of composite thin-walled members. In: Loughlan J (ed) Proceedings of the 4th International Conference on Thin-Walled Structures. IOP Publishing Ltd, Bristol

Silvestre N, Camotim D (2012) A shear deformable generalized beam theory for the analysis of thin-walled composite members. J Eng Mech 139(8):1010-1024

Tong GS, Yan XX, Zhang L (2005) Warping and bimoment transmissiion through diagonally stiffened beam-to-column joints. J Constr Steel Res 61:749-763

Vlasov VZ (1958) Thin-walled elastic bars. Fizmatgiz, Moscow (Russian - English translation: Israel Program forScientific Translation, Jerusalém, 1961)

Publisher's Note Springer Nature remains neutral with regard to jurisdictional claims in published maps and institutional affiliations. 